\title{
Statistics of the MLE and Approximate Upper and Lower Bounds-Part II: Threshold Computation and Optimal Pulse Design for TOA Estimation
}

\author{
Achraf Mallat, Member, IEEE, Sinan Gezici, Senior Member, IEEE, Davide Dardari, Senior Member, IEEE, and
} Luc Vandendorpe, Fellow, IEEE

\begin{abstract}
Threshold and ambiguity phenomena are studied in Part I of this paper where approximations for the mean-squared error (MSE) of the maximum-likelihood estimator are proposed using the method of interval estimation (MIE), and where approximate upper and lower bounds are derived. In this part, we consider time-of-arrival estimation and we employ the MIE to derive closed-form expressions of the begin-ambiguity, end-ambiguity and asymptotic signal-to-noise ratio (SNR) thresholds with respect to some features of the transmitted signal. Both baseband and passband pulses are considered. We prove that the begin-ambiguity threshold depends only on the shape of the envelope of the ACR, whereas the end-ambiguity and asymptotic thresholds only on the shape of the ACR. We exploit the results on the begin-ambiguity and asymptotic thresholds to optimize, with respect to the available $S N R$, the pulse that achieves the minimum attainable MSE. The results of this paper are valid for various estimation problems.
\end{abstract}

Index Terms-Maximum likelihood estimator, mean-squarederror, nonlinear estimation, optimal signal design, signal-to-noise ratio, threshold and ambiguity phenomena, time-of-arrival.

\section{INTRODUCTION}

$\mathbf{N}$ ONLINEAR deterministic parameter estimation is subject to the threshold effect [2]-[9]. Due to this effect the signal-to-noise ratio (SNR) axis can be split into three regions (see Fig. 1(a) in Part I of this work [1]):

1) A priori region: Region in which the estimator becomes uniformly distributed in the a priori domain.

Manuscript received October 20, 2013; revised April 07, 2014 and August 16, 2014; accepted August 26, 2014. Date of publication September 08, 2014; date of current version October 07, 2014. The associate editor coordinating the review of this manuscript and approving it for publication was Dr. Petr Tichavsky. This work has been supported in part by the Belgian IAP network Bestcom funded by Belspo, the PEGASO project funded by the Walloon region Skywin pole, the FNRS, and the European Commission in the framework of the FP7 Network of Excellence in Wireless COMmunications NEWCOM \# (Contract no. 318306). S. Gezici's research was supported in part by the Young Scientists Award Programme of Turkish Academy of Sciences (TUBA-GEBIP 2013).

A. Mallat and L. Vandendorpe are with the Institute for Information and Communication Technologies, Electronics and Applied Mathematics (ICTEAM Institute), Université Catholique de Louvain, Louvain-la-Neuve 1348, Belgium (e-mail: Achraf.Mallat@uclouvain.be; Luc.Vandendorpe@uclouvain.be).

S. Gezici is with the Department of Electrical and Electronics Engineering, Bilkent University, Ankara 06800, Turkey (e-mail: gezici@ee.bilkent.edu.tr).

D. Dardari is with DEI, CNIT, University of Bologna, Bologna 40126, Italy (e-mail: davide.dardari@unibo.it).

Color versions of one or more of the figures in this paper are available online at http://ieeexplore.ieee.org.

Digital Object Identifier 10.1109/TSP.2014.2355776
2) Threshold region: Region of transition between the a priori and asymptotic regions.

3) Asymptotic region: Region in which an asymptotically efficient estimator, such as the maximum likelihood estimator (MLE), achieves the Cramer-Rao lower bound (CRLB). Otherwise, the estimator achieves its own asymptotic mean-squared-error (MSE) (e.g, MLE with random signals and finite snapshots [10], [11]).

When the autocorrelation (ACR) with respect to (w.r.t.) the unknown parameter is oscillating, five regions can be identified (as can be seen in Fig. 1(b) in Part I [1]): 1) the a priori region, 2) the a priori-ambiguity transition region, 3 ) the ambiguity region where the envelope CRLB (ECRLB) is achieved [1], 4) the ambiguity-asymptotic transition region, and 5) the asymptotic region.

As the evaluation of the statistics of most estimators such as the MLE is often unattainable in the threshold region, many lower bounds have been proposed [12], [13] for both deterministic (the unknown parameter has only one possible value) and Bayesian (the unknown parameter follows a given a priori distribution) estimation in order to be used as benchmarks and to describe the behavior of an estimator in that region.

Threshold computation is considered in [4], [5] where the $a$ priori, begin-ambiguity, end-ambiguity and asymptotic thresholds are computed based on the Ziv-Zakai lower bound (ZZLB); the ZZLB evaluates accurately the asymptotic threshold and detects roughly the ambiguity region. Thresholds are also computed in [6], [7] using the Barankin lower bound (BLB); the obtained thresholds are much smaller than the true ones. Closedform expressions of the asymptotic threshold are derived in [14] for frequency estimation and in [15] for angle estimation by employing the method of interval estimation (MIE). The method in [14] is based on the MSE approximation (MSEA) in [16] and is valid for cardinal sine ACRs only, whereas that in [15] is based on the probability of non-ambiguity and can be used with any ACR shape. The approaches in [14], [15] are discussed in details and compared to our approach in Section IV.

Optimal power allocation for multicarrier systems with interference is considered in [17]; the approach followed therein minimizes the CRLB for TOA estimation without taking into account the threshold and ambiguity effects. Optimal pulse design for TOA estimation is studied in [18] based on the BLB; the authors study the reduction of the asymptotic threshold by considering different ACR shapes. The optimization of the time- 
bandwidth product for frequency estimation is investigated in [19] based on the MIE. The approach in [19] is discussed and compared to ours in Section VI.

In Part I of this work [1], an approximate upper bound and various MSEAs for the MLE are proposed by using the MIE [12], [15], [16], [18]-[26]. Some approximate lower bounds (ALB) are proposed as well by employing the binary detection principle first used by Ziv and Zakai [2].

In Part II (current paper), we have three main contributions. The first contribution is that we utilize an MIE-MSEA (proposed later in Section III-A) to derive analytic expressions of the begin-ambiguity, end-ambiguity and asymptotic thresholds. The obtained thresholds are very accurate (in particular the endambiguity and asymptotic thresholds of oscillating ACRs). To the best of our knowledge, our approach is the first utilizing an MIE-based MSEA (very accurate approximation) and that can be used with any ACR shape. The equations established in this paper are obtained by considering TOA estimation. However, our method can be applied on any estimation problem satisfying the system model of Part I.

The second contribution is that we demonstrate some properties of the thresholds. We prove that the begin-ambiguity threshold only depends on the shape of the ACR envelope (e.g., cardinal sine, Gaussian, raised cosine with fixed roll-off) regardless of other parameters (e.g., a priori domain, bandwidth, mean frequency), and the end-ambiguity and asymptotic thresholds only depend on the ACR shape (which can be described by the envelope shape and the mean frequency to bandwidth ratio, together) regardless of other parameters (e.g, the bandwidth and the mean frequency if their ratio is constant). The thresholds of the different SNR regions are also evaluated numerically using an MSEA and two ALBs (derived in Part I). We show that the a priori threshold depends on both the $a$ priori domain and the shape of the ACR envelope.

The third contribution is that we make use of the obtained results about the thresholds to propose a method to optimize, w.r.t. the available SNR, the spectrum of the transmitted pulse in order to achieve the minimum attainable MSE. The proposed method is very simple and very accurate. To the best of our knowledge, this is the first optimization problem addressing the minimization of the MSE subject to the threshold and ambiguity phenomena.

The rest of the paper is organized as follows. In Section II we describe the system model. In Section III we introduce some MIE-based MSEAs and ALBs. In Section IV we consider the numerical and analytical computation of the thresholds and analyze their properties. In Section V we present and discuss some numerical results about the thresholds when baseband and passband pulses are employed. In Section VI we derive the MLE of the SNR and propose a method to optimize the spectrum of the transmitted pulse w.r.t. the available SNR.

\section{SYSTEM MODEL}

In this section we describe our system model. Let $s(t)$ be the transmitted signal, $\alpha$ and $\Theta$ the positive gain and the time delay introduced by an additive white Gaussian noise (AWGN) channel, and $\tilde{w}(t)$ the noise with two-sided power spectral density (PSD) of $\frac{N_{0}}{2}$. We can write the received signal as:

$$
r(t)=\alpha s(t-\Theta)+\tilde{w}(t) .
$$

We assume that $\Theta$ is deterministic with $D_{\Theta}=\left[\Theta_{1}, \Theta_{2}\right]$ representing its a priori domain.

From Part I, the MLE of $\Theta$ is given by

$$
\hat{\Theta}=\underset{\theta}{\operatorname{argmax}}\left\{X_{r, s}(\theta)\right\}
$$

where $X_{r, s}(\theta)=\alpha R_{s}(\theta-\Theta)+w(\theta)$ is the CCR of $r(t)$ and $s(t)$ with $R_{s}(\theta)=\int_{-\infty}^{+\infty} s(t) s(t-\theta) d t$ being the ACR of $s(t)$ and $w(\theta)=\int_{-\infty}^{+\infty} r(t) \tilde{w}(t-\theta) d t$ a zero-mean colored Gaussian noise of covariance $C_{w}(\theta)=\frac{N_{0}}{2} R_{s}(\theta)$.

From Part I, we can express the CRLB, the ECRLB and the maximum MSE of $\Theta$ as:

$$
\begin{aligned}
c & =\frac{1}{\rho \beta_{s}^{2}} \\
c_{e} & =\frac{1}{\rho \beta_{e}^{2}} \\
e_{U} & =\frac{\left(\Theta_{2}-\Theta_{1}\right)^{2}}{12}+\left[\Theta-\frac{\Theta_{1}+\Theta_{2}}{2}\right]^{2}
\end{aligned}
$$

where $\rho=\frac{\alpha^{2} E_{s}}{N_{0} / 2}$ denotes the SNR, and $\beta_{s}^{2}$ and $\beta_{e}^{2}$ stand for the mean quadratic bandwidth (MQBW) and the envelope MQBW (EMQBW) of $s(t)$, respectively. We have:

$$
\beta_{s}^{2}=-\frac{\ddot{R}_{s}(0)}{E_{s}}=\beta_{e}^{2}+4 \pi^{2} f_{c}^{2} \approx 4 \pi^{2} f_{c}^{2}
$$

where $\ddot{R}_{s}(\theta)$ denotes the second derivative of $R_{s}(\theta), E_{s}=$ $\int_{-\infty}^{+\infty} s^{2}(t) d t$ and $f_{c}=\frac{\int_{0}^{+\infty} f\left|\mathcal{F}_{s}(f)\right|^{2} d f}{\int_{0}^{+\infty}\left|\mathcal{F}_{s}(f)\right|^{2} d f}$ represent the energy and the mean frequency of $s(t)$, with $\mathcal{F}_{s}(f)$ being the Fourier transform of $s(t)$.

We have seen in Part I, that for a signal occupying the whole band from 3.1 to $10.6 \mathrm{GHz}^{1}\left(f_{c}=6.85 \mathrm{GHz}\right.$, bandwidth $B=$ $7.5 \mathrm{GHz}$ ), we have $\beta_{e}^{2}=\frac{\pi^{2} B^{2}}{3} \approx \frac{4 \pi^{2} f_{c}^{2}}{10}$, so $c \approx \frac{c_{e}}{11}$. Therefore, the estimation performance seriously deteriorates if the ECRLB is achieved instead of the CRLB due to ambiguity.

As $\beta_{e}^{2} \ll 4 \pi^{2} f_{c}^{2}$, the super accuracy associated with $c$ is mainly due to the mean frequency $f_{c}$. To benefit from this super accuracy at sufficiently high SNRs, the sufficient condition to satisfy is that the phase of the transmitted signal should not be modified across the channel (e.g, due to fading), regardless whether the signal is pure impulse-radio UWB (carrier-less), carrier-modulated with known phase (e.g, in monostatic radar), or carrier-modulated with unknown phase (e.g, in most communication systems). With the latter, we have to use the time difference of arrival (TDOA) technique.

\section{MSEAS AND ALBS}

In this section we introduce some MSEAs and ALBs that will be used later in Sections IV and V to compute the thresholds.

\section{A. MIE-Based MSEAs}

We have seen in Part I that by splitting the a priori domain of $\Theta$ into $N$ intervals $D_{n}=\left[d_{n}, d_{n+1}\right),\left(n=n_{1}, \ldots, n_{N}\right)$, $\left(n_{1} \leq 0, n_{N} \geq 0\right)$, we can write the MSE of $\hat{\Theta}$ as:

$$
e(\rho)=\sum_{n=n_{1}}^{n_{N}} P_{n}\left[\left(\Theta-\mu_{n}\right)^{2}+\sigma_{n}^{2}\right]
$$

\footnotetext{
${ }^{1}$ The ultra wideband (UWB) spectrum authorized for unlicensed use by the
} US federal commission of communications in May 2002 [27]. 
where $P_{n}=\mathbb{P}\left\{\hat{\Theta} \in D_{n}\right\}$ denotes the interval probability, and $\mu_{n}=\mathbb{E}\left\{\hat{\Theta}_{n}\right\}$ and $\sigma_{n}^{2}=\mathbb{E}\left\{\left(\hat{\Theta}_{n}-\mu_{n}\right)^{2}\right\}$ represent, respectively, the mean and the variance of the interval MLE $\hat{\Theta}_{n}=\hat{\Theta} \mid \hat{\Theta} \in$ $D_{n}(\mathbb{P}$ and $\mathbb{E}$ stand for the probability and expectation operators). For oscillating (resp. non-oscillating) ACRs, we consider an interval around each local maximum (resp. split $D_{\Theta}$ into $N$ equal duration intervals); $D_{0}$ always contains the maximum of the ACR.

Different approximations of $P_{n}, \mu_{n}$ and $\sigma_{n}^{2}$ were proposed in Part I. Below, we only present the approximations that will be used later in this paper for the numerical and the analytic evaluation of the thresholds.

1) An MSEA for Numerical Threshold Computation: We present in this paragraph the MSEA

$$
e_{\text {num }}(\rho)
$$

based on (5) and that we will use later in Section V for the numerical evaluation of the different thresholds; $e_{\text {num }}(\rho)$ is the most accurate approximation proposed in Part I.

For both oscillating and non-oscillating ACRs, $P_{n}$ in (5) is approximated by $P_{n}^{(1)}=\operatorname{Genz} \operatorname{Algo}\left(\theta_{n_{1}}, \ldots, \theta_{n_{N}}\right)$ where GenzAlgo denotes one of Genz's algorithms written based on [28]-[31] to compute the multivariate normal probability with integration region specified by a set of linear inequalities (see Part I for more details), and $\theta_{n}$ represents a testpoint in $D_{n}$; $\theta_{n}$ is selected as the abscissa of the $n$th local maximum (resp. the center of $D_{n}$ ) for oscillating (resp. non-oscillating) ACRs; $\theta_{0}=\Theta$ (abscissa of the maximum) for both ACR types.

For oscillating (resp. non-oscillating) ACRs, $\mu_{n}$ and $\sigma_{n}^{2}$ are approximated by $\mu_{n, 1, o}=\theta_{n}$ and $\sigma_{n, 1, o}^{2}=$ $\min \left\{c \frac{\ddot{R}_{0}^{2}}{\ddot{R}_{n}^{2}}, \sigma_{n, U}^{2}\right\}$ (resp. $\mu_{n, 1, c}=d_{n} P\left\{d_{n}\right\}+d_{n+1} P\left\{d_{n+1}\right\}$ and $\left.\sigma_{n, 1, c}^{2}=\min \left\{\sigma_{n, B}^{2}, \sigma_{n, U}^{2}\right\}\right)$ where $\ddot{R}_{n}=\left.\frac{d^{2} R_{s}(\theta)}{d \theta^{2}}\right|_{\theta=\theta_{n}}$, $\sigma_{n, U}^{2}=\frac{\left(d_{n+1}-d_{n}\right)^{2}}{12}, P\left\{d_{n}\right\}=Q\left(\sqrt{\rho} \frac{\dot{R}_{n}}{E_{s} \beta_{s}}\right)$ and $\sigma_{n, B}^{2}=\mathbb{P}\left\{d_{n}\right\}\left(1-\mathbb{P}\left\{d_{n}\right\}\right)\left(d_{n+1}-d_{n}\right)^{2}$, with $Q(y)=$ $\frac{1}{\sqrt{2 \pi}} \int_{y}^{\infty} e^{-\frac{\xi^{2}}{2}} d \xi$ being the $\mathrm{Q}$ function and $\dot{R}_{n}=\left.\frac{d R_{s}(\theta)}{d \theta}\right|_{\theta=\theta_{n}}$.

2) An MSEA for Analytic Threshold Computation: The MSEA $e_{\text {ana }}(\rho)$ proposed in this paragraph will be used later in Section IV-B to express analytically the end-ambiguity and asymptotic thresholds; $e_{\text {ana }}(\rho)$ employs the probability upper bound proposed by McAulay in [18]. It evaluates the achieved MSE in the intervals $D_{-1}, D_{0}$ and $D_{1}$, which means that the SNR is supposed to be relatively high.

By approximating $\mu_{n}$ in (5) by $\theta_{n}$, approximating $\sigma_{0}^{2}$ by $c$, neglecting $\sigma_{ \pm 1}^{2}\left(\sigma_{ \pm 1}^{2} \ll\left(\Theta-\mu_{ \pm 1}\right)^{2}\right)$, taking $\theta_{0}=\Theta$ and $\theta_{ \pm 1}=\Theta \pm \Delta$ with $\Delta=\frac{1}{f_{c}} \approx \frac{2 \pi}{\beta_{s}}$ for oscillating ACRs $\left(\theta_{ \pm 1}\right.$ are the approximate abscissa of the two local maxima around the global one) and $\Delta=\frac{\pi}{4 \beta_{s}}$ for non-oscillating $\operatorname{ACRs}\left(\theta_{ \pm 1}\right.$ are empirically chosen, see Section V-B in Part I for more details), and adopting the McAulay probability upper bounds $P_{0}^{(2)}=1$ and $P_{ \pm 1}^{(2)}=Q\left(\sqrt{\frac{\rho}{2}[1-R(\Delta)]}\right)$ with $R(\theta)=\frac{R_{s}(\theta)}{E_{s}}$ denoting the normalized ACR, $e_{\text {ana }}(\rho)$ becomes

$$
e_{\mathrm{ana}}(\rho)=c+2 \Delta^{2} Q\left(\sqrt{\frac{\rho}{2}[1-R(\Delta)]}\right) .
$$

Let us now explain why $e_{\text {ana }}(\rho)$ is appropriate for the evaluation of the end-ambiguity and asymptotic thresholds. Assume for the moment that the CRLB is achieved (i.e., the SNR is sufficiently high). In the course of decreasing the SNR, the threshold (resp. ambiguity) region begins for non-oscillating (resp. oscillating) ACRs when the estimates of the unknown parameter start to spread along the ACR (resp. the local maxima of the ACR) instead of falling in the vicinity of the maximum (resp. global maximum). Therefore, the estimates only fall at the end of the threshold and ambiguity regions (if we start from low SNRs) in the interval $D_{0}$ and the intervals $D_{-1}$ and $D_{1}$ (at the left and the right of $D_{0}$ ) so the achieved MSE can be approximated using $e_{\text {ana }}(\rho)$.

\section{B. Binary Detection Based ALBs}

By using the principle of binary detection, we have derived in Part I the following ALBs $(i=1,2)$ :

$$
\begin{aligned}
z_{i} & =\int_{0}^{\epsilon_{i}} \xi Q\left(\sqrt{\frac{\rho}{2}[1-R(\xi)]}\right) d \xi \\
b_{i} & =\int_{0}^{\epsilon_{i}} \xi V\left\{Q\left(\sqrt{\frac{\rho}{2}[1-R(\xi)]}\right)\right\} d \xi
\end{aligned}
$$

where $\epsilon_{1}=\min \left\{\Theta-\Theta_{1}, 2\left(\Theta_{2}-\Theta\right)\right\}$ and $\epsilon_{2}=\min \left\{\Theta_{2}-\right.$ $\left.\Theta, 2\left(\Theta-\Theta_{1}\right)\right\} ; V\{f(\xi)\}=\max \{f(\zeta \geq \xi)\}$ denotes the valley-filling function. We have seen in Part I that $z_{i}$ and $b_{i}$ are very tight and that $b_{i}$ is tighter than $z_{i} ; z_{1}$ and $b_{1}$ are, respectively, tighter than $z_{2}$ and $b_{2}$ when $\theta_{0}-\Theta_{1}>\Theta_{2}-\theta_{0}$.

\section{THRESHOLD COMPUTATION}

We consider in this section the computation of the thresholds of the different SNR regions w.r.t. some features of the transmitted signal.

Similarly to Part I, we define the a priori $\rho_{p r}$, begin-ambiguity $\rho_{a m 1}$, end-ambiguity $\rho_{a m 2}$ and asymptotic $\rho_{a s}$ thresholds as [5]:

$$
\begin{aligned}
\rho_{p r} & =\rho: e(\rho)=\alpha_{p r} e_{U} \\
\rho_{a m 1} & =\rho: e(\rho)=\alpha_{a m 1} c_{e} \\
\rho_{a m 2} & =\rho: e(\rho)=\alpha_{a m 2} c_{e} \\
\rho_{a s} & =\rho: e(\rho)=\alpha_{a s} c .
\end{aligned}
$$

We take $\alpha_{p r}=0.5, \alpha_{a m 1}=2, \alpha_{a m 2}=0.5$ and $\alpha_{a s}=1.1$.

The considered features of the transmitted signal are the $a$ priori time bandwidth product (ATBW) and the inverse fractional bandwidth (IFBW) defined as:

$$
\begin{aligned}
& \gamma=T B \\
& \lambda=\frac{f_{c}}{B}
\end{aligned}
$$

where $T=\Theta_{2}-\Theta_{1}$ (a priori time) is the width of the a priori domain of $\Theta$ and $B$ the bandwidth of the transmitted signal.

In Section IV-A, we consider the numerical calculation of the thresholds. We derive in Section IV-B analytic expressions of the begin-ambiguity, end-ambiguity and asymptotic thresholds, and discuss in Section IV-C the properties of the thresholds obtained in Section IV-B.

\section{A. Numerical Computation}

As mentioned above we consider here the numerical computation of the thresholds. To find $\rho_{p r}, \rho_{a m 1}, \rho_{a m 2}$ and $\rho_{a s}$ w.r.t. 
$\gamma$ (resp. $\lambda$ ) numerically, we vary $\gamma$ (resp. $\lambda$ ) by fixing $T$ (resp. $f_{c}$ ) and varying $B$ (or vice versa) and compute for each value of $\gamma$ (resp. $\lambda$ ) the achieved MSE along the SNR axis. Then, the thresholds are then obtained by making use of (10)-(13).

Theoretically, the thresholds should be computed from the MSE achieved in practice. As the exact expression of the MSE is not obtainable in most estimation problems, the thresholds can be calculated using a MSEA, an upper bound or a lower bound. In Section $\mathrm{V}$, the a priori, begin-ambiguity and end-ambiguity thresholds are computed numerically using the MSEA $e_{\text {num }}(\rho)$ in (6). The asymptotic threshold is computed using $e_{\text {num }}(\rho)$ and the ALBs $z_{i}$ in (8) and $b_{i}$ in (9).

\section{B. Analytic Expressions of the Begin-Ambiguity, \\ End-Ambiguity and Asymptotic Thresholds}

In this subsection, we derive analytic expressions of the begin-ambiguity, end-ambiguity and asymptotic thresholds by making use of the MSEA $e_{\text {ana }}(\rho)$ in (7).

1) Asymptotic Threshold for Oscillating and Non-Oscillating ACRs: Let:

$$
G(\rho)=\rho Q\left(\sqrt{\frac{\rho}{2}[1-R(\Delta)]}\right)
$$

Using (1), (7) and (16) we can write from the asymptotic threshold definition in (13):

$$
G\left(\rho_{a s}\right)=G_{a s}
$$

where

$$
G_{a s}=\frac{\alpha_{a s}-1}{2 \Delta^{2} \beta_{s}^{2}}
$$

denotes a constant; $\rho_{a s}$ is the solution of (17).

To find an analytic expression of $\rho_{a s}$ we consider the following approximation of the $\mathrm{Q}$ function

$$
Q(\xi) \approx \frac{1}{\xi} \frac{1}{\sqrt{2 \pi}} e^{-\frac{\xi^{2}}{2}}, \xi \gg 1
$$

obtained from the inequality $\left(\frac{1}{\xi}-\frac{1}{\xi^{3}}\right) \frac{1}{\sqrt{2 \pi}} e^{-\frac{\xi^{2}}{2}}<Q(\xi)<$ $\frac{1}{\xi} \frac{1}{\sqrt{2 \pi}} e^{-\frac{\xi^{2}}{2}}, \xi>0$ in [22, pp. 83]. Let:

$$
H(\rho)=-\frac{\rho[1-R(\Delta)]}{2}
$$

From (18), (19) and (20), we can write (17) as:

$$
H\left(\rho_{a s}\right) e^{H\left(\rho_{a s}\right)}=H_{a s}
$$

with

$$
H_{a s}=-\frac{\pi G_{a s}^{2}[1-R(\Delta)]}{2}=-\frac{\pi\left(\alpha_{a s}-1\right)^{2}[1-R(\Delta)]}{8 \Delta^{4} \beta_{s}^{4}}
$$

so the asymptotic threshold in (21) can be expressed as:

$$
\rho_{a s}=\frac{-2 W_{-1}\left(H_{a s}\right)}{1-R(\Delta)}
$$

where $W_{-1}(\xi)$ denotes the branch " -1 " (because $H_{a s}$ is negative) of the Lambert $\mathrm{W}$ function defined as a solution (more than one solution may exist) of the equation $W e^{W}=\xi$. Like the other non-elementary functions (e.g, Q function, error function), the Lambert $\mathrm{W}$ function has Taylor series expansion and can be computed recursively; it is also implemented in
MATLAB; hence, the solution in (20) can be considered as an analytic solution since it can directly be obtained.

We recall that in the evaluation of $G_{a s}$ in (18), $H_{a s}$ in (22) and $\rho_{a s}$ in (23), we take $\Delta=\frac{\pi}{4 \beta_{s}}$ for non-oscillating ACRs and $\Delta \approx \frac{1}{f_{c}} \approx \frac{2 \pi}{\beta_{s}}$ for oscillating ACRs.

2) End-Ambiguity Threshold for Oscillating ACRs: From the end-ambiguity threshold definition in (12) we can write using (1), (2), (4), (7) and (16):

$$
G\left(\rho_{a m 2}\right)=G_{a m 2}
$$

where

$$
G_{a m 2}=\frac{1}{2 \Delta^{2}}\left(\frac{\alpha_{a m 2}}{\beta_{e}^{2}}-\frac{1}{\beta_{s}^{2}}\right) \approx \frac{\alpha_{a m 2}}{2 \Delta^{2} \beta_{e}^{2}} .
$$

Using (19), (20) and (25), we can write (24) as:

$$
H\left(\rho_{a m 2}\right) e^{H\left(\rho_{a m 2}\right)}=H_{a m 2}
$$

where

$$
H_{a m 2}=-\frac{\pi G_{a m 2}^{2}[1-R(\Delta)]}{2} \approx-\frac{\pi \alpha_{a m 2}^{2}[1-R(\Delta)]}{8 \Delta^{4} \beta_{e}^{4}}
$$

so the end-ambiguity threshold in (26) can be expressed as:

$$
\rho_{a m 2}=\frac{-2 W_{-1}\left(H_{a m 2}\right)}{1-R(\Delta)} .
$$

We recall that in the evaluation of $G_{a m 2}$ in (25), $H_{a m 2}$ in (27) and $\rho_{a m 2}$ in (28), we take $\Delta \approx \frac{1}{f_{c}} \approx \frac{2 \pi}{\beta_{s}}$.

3) Begin-Ambiguity Threshold for Oscillating ACRs: To compute the begin-ambiguity threshold, we cannot employ the MSEA in (7) because the estimates fall now, not only in $D_{-1}$, $D_{0}$ and $D_{1}$, but around all the local maxima in the vicinity of the maximum of the envelope of the ACR. Therefore, by considering the envelope $e_{R}(\theta)$ of the normalized ACR $R(\theta)$ instead of $R(\theta)$ itself, and the ECRLB $c_{e}$ in (2) instead of the CRLB $c$ in (1), we can approximate the MSE in the vicinity of the maximum of $e_{R}(\theta)$ by:

$$
e_{\mathrm{ana}, e}(\rho) \approx c_{e}+2 \Delta^{2} Q\left(\sqrt{\frac{\rho}{2}\left[1-e_{R}(\Delta)\right]}\right)
$$

where, similarly to the case of non-oscillating ACRs, we take $\Delta=\frac{\pi}{4 \beta}\left(\beta_{s}\right.$ is replaced by $\beta_{e}$ because the EMQBW is equal to the MQBW of the envelope). Let:

$$
\begin{aligned}
& G_{e}(\rho)=\rho Q\left(\sqrt{\frac{\rho}{2}\left[1-e_{R}(\Delta)\right]}\right) \\
& H_{e}(\rho)=-\frac{\rho\left[1-e_{R}(\Delta)\right]}{2}
\end{aligned}
$$

From (2), (29), (30) and (31) we can write the definition of the begin-ambiguity threshold in (11) as:

$$
G_{e}\left(\rho_{a m 1}\right)=G_{a m 1}
$$

where

$$
G_{a m 1}=\frac{\alpha_{a m 1}-1}{2 \Delta^{2} \beta_{e}^{2}} .
$$

Using (19), (32) becomes:

$$
H_{e}\left(\rho_{a m 1}\right) e^{H_{e}\left(\rho_{a m 1}\right)}=H_{a m 1}
$$


where

$$
H_{a m 1}=-\frac{\pi G_{a m 1}^{2}\left[1-e_{R}(\Delta)\right]}{2}=-\frac{\pi\left(\alpha_{a m 1}-1\right)^{2}\left[1-e_{R}(\Delta)\right]}{8 \Delta^{4} \beta_{e}^{4}}
$$

so we can express the begin-ambiguity threshold from (34) as:

$$
\rho_{a m 1}=\frac{-2 W_{-1}\left(H_{a m 1}\right)}{1-e_{R}(\Delta)} .
$$

We recall that in the evaluation of $G_{a m 1}$ in (33), $H_{a m 1}$ in (35) and $\rho_{a m 1}$ in (36), we take $\Delta=\frac{\pi}{4 \beta_{e}}$.

4) About the End-Ambiguity and Asymptotic Thresholds for Oscillating ACRs: Note that in the computation of the end-ambiguity and asymptotic thresholds for oscillating ACRs, $R(\Delta)$ can be replaced by $e_{R}(\Delta)$ because $\theta_{ \pm 1}$ in (7) are the abscissa of two local maxima of $R(\theta-\Theta)$ (the local maxima are located on the envelope). Therefore, $\rho_{a s}$ in (23) and $\rho_{a m 2}$ in (28) can be expressed as:

$$
\begin{gathered}
\rho_{a s}=\frac{-2 W_{-1}\left(H_{a s}\right)}{1-e_{R}(\Delta)} \\
\rho_{a m 2}=\frac{-2 W_{-1}\left(H_{a m 2}\right)}{1-e_{R}(\Delta)}
\end{gathered}
$$

where

$$
\begin{aligned}
H_{a s} & =-\frac{\pi\left(\alpha_{a s}-1\right)^{2}\left[1-e_{R}(\Delta)\right]}{8 \Delta^{4} \beta_{s}^{4}} \\
H_{a m 2} & =-\frac{\pi \alpha_{a m 2}^{2}\left[1-e_{R}(\Delta)\right]}{8 \Delta^{4} \beta_{e}^{4}} .
\end{aligned}
$$

By using (37) and (38) instead of (23) and (28), we highly simplify the calculation of the thresholds. In fact, if we want to compute the thresholds of a passband pulse (i.e., pulse modulated by carrier) w.r.t. the IFBW $\lambda$ in (15), then instead of generating the normalized ACR $R(\theta)$ for each value of $\lambda$, we just compute the normalized ACR envelope $e_{R}(\theta)$ once and evaluate $R(\Delta)=e_{R}(\Delta)$ by varying $\Delta$ w.r.t. $\lambda$.

\section{Threshold Properties}

In this subsection we prove that for a baseband (i.e., unmodulated) pulse that can be written as (e.g, Gaussian, cardinal sine and raised cosine pulses):

$$
w_{B}(t)=w_{1}\left(t^{\prime}\right), t^{\prime}=B t
$$

with $B$ denoting the bandwidth, the asymptotic threshold only depends on the shape $w_{1}(t)$ (i.e., independent of $B$ ) (e.g, constant for Gaussian and cardinal sine pulses, and function of the roll-off factor for raised cosine pulses), and that for the passband pulse

$$
\begin{aligned}
w_{B, f_{c}}(t) & =w_{B}(t) \cos \left(2 \pi f_{c} t\right) \\
& =w_{1}\left(t^{\prime}\right) \cos \left(2 \pi \lambda t^{\prime}\right), \quad t^{\prime}=B t
\end{aligned}
$$

with $f_{c}$ denoting the carrier frequency, the begin-ambiguity threshold only depends on the shape $w_{1}(t)$ of the envelope $w_{B}(t)$ of $w_{B, f_{c}}(t)$ (i.e., independent of $B, f_{c}$ and the IFBW $\lambda$ ), whereas the end-ambiguity and asymptotic thresholds are functions of the shape $w_{1}(t)$ and the IFBW $\lambda$ in (15) (i.e., independent of the values taken by $B$ and $f_{c}$ separately). This is equivalent to saying that the begin-ambiguity threshold is only function of the shape of the envelope of the signal, whereas the end-ambiguity and asymptotic thresholds are only functions of the shape of the signal itself, regardless of any other parameters like the bandwidth and the carrier frequency.

1) Asymptotic Threshold for Baseband Pulses: Let us prove that the asymptotic threshold in (23) of the pulse $w_{B}(t)$ in (41) is independent of $B$. From (41) we can write the normalized ACR $R_{B}(\theta)$ of $w_{B}(t)$ as:

$$
\begin{aligned}
R_{B}(\theta) & =\frac{\int_{-\infty}^{+\infty} w_{B}(t) w_{B}(t-\theta) d t}{\int_{-\infty}^{+\infty} w_{B}^{2}(t) d t}=\frac{\int_{-\infty}^{+\infty} w_{1}\left(t^{\prime}\right) w_{1}\left(t^{\prime}-\theta^{\prime}\right) d t^{\prime}}{\int_{-\infty}^{+\infty} w_{1}^{2}\left(t^{\prime}\right) d t^{\prime}} \\
& =R_{1}\left(\theta^{\prime}\right), \theta^{\prime}=B \theta
\end{aligned}
$$

where $R_{1}(\theta)$ denotes the normalized ACR of $w_{1}(t)$, and the MQBW $\beta_{B}^{2}$ of $w_{B}(t)$ using (4) and (43) as:

$$
\beta_{B}^{2}=-\left.\frac{d^{2} R_{B}(\theta)}{d \theta^{2}}\right|_{\theta=0}=-\left.B^{2} \frac{d^{2} R_{1}\left(\theta^{\prime}\right)}{d \theta^{\prime 2}}\right|_{\theta^{\prime}=0}=B^{2} \beta_{1}^{2}
$$

where $\beta_{1}^{2}=-\ddot{R}_{1}(0)$ denotes the MQBW of $w_{1}(t)$ (unitary MQBW, i.e., MQBW per a bandwidth of $B=1 \mathrm{~Hz}$ ). Note that $R_{B}(\theta)$ and $\beta_{B}$ used here are, respectively, equivalent to $R(\theta)$ and $\beta_{s}$ used in Section IV-B. As $\Delta=\frac{\pi}{4 \beta_{s}}=\frac{\pi}{4 \beta_{B}}$ for non-oscillating ACRs, we can write $R(\Delta)$ and $H_{a s}$ in (23) from (43) and (44) as:

$$
\begin{aligned}
R(\Delta) & =R_{B}\left(\frac{\pi}{4 \beta_{B}}\right)=R_{B}\left(\frac{\pi}{4 B \beta_{1}}\right)=R_{1}\left(\frac{\pi}{4 \beta_{1}}\right) \\
H_{a s} & =-\frac{32\left(\alpha_{a s}-1\right)^{2}\left[1-R_{1}\left(\frac{\pi}{4 \beta_{1}}\right)\right]}{\pi^{3}} .
\end{aligned}
$$

We can see that both $R(\Delta)$ and $H_{a s}$ are independent of $B$. Hence, for the pulse in (41) the asymptotic threshold is independent of $B$; it depends only on the shape of the normalized ACR $R_{B}(\theta)$ determined by $R_{1}(\theta)$.

2) Begin-Ambiguity Threshold for Passband Pulses: Let us prove that the begin-ambiguity threshold in (36) of the pulse $w_{B, f_{c}}(t)$ in (42) is independent of $B$ and $f_{c}$. The envelope $e_{R_{B, f_{c}}}(\theta)$ of the normalized ACR $R_{B, f_{c}}(\theta)$ of $w_{B, f_{c}}(t)$ and the EMQBW $\beta_{e, B, f_{c}}^{2}$ of $w_{B, f_{c}}(t)$ can be written from (42), (43) and (44) as:

$$
\begin{aligned}
e_{R_{B, f_{c}}}(\theta) & =R_{B}(\theta)=R_{1}\left(\theta^{\prime}\right), \theta^{\prime}=B \theta \\
\beta_{e, B, f_{c}}^{2} & =\beta_{B}^{2}=B^{2} \beta_{1}^{2} .
\end{aligned}
$$

Note that $e_{R_{B, f_{c}}}(\theta)$ and $\beta_{e, B, f_{c}}^{2}$ used here are, respectively, equivalent to $e_{R}(\theta)$ and $\beta_{e}$ used in Section IV-B. As $\Delta=\frac{\pi}{4 \beta_{\epsilon}}=\frac{\pi}{4 \beta_{e, B, f_{c}}}$ for the begin-ambiguity threshold, we can write $e_{R}(\Delta)$ and $H_{a m 1}$ in (36) using (45) and (46) as:

$$
\begin{aligned}
e_{R}(\Delta) & =R_{B}\left(\frac{\pi}{4 \beta_{B}}\right)=R_{1}\left(\frac{\pi}{4 \beta_{1}}\right) \\
H_{a m 1} & =-\frac{32\left(\alpha_{a m 1}-1\right)^{2}\left[1-R_{1}\left(\frac{\pi}{4 \beta_{1}}\right)\right]}{\pi^{3}} .
\end{aligned}
$$

Both $e_{R}(\Delta)$ and $H_{a m 1}$ are independent of $B$ and $f_{c}$. Hence, for the pulse in (42) the begin-ambiguity threshold is independent of $B$ and $f_{c}$; it only depends on the shape $R_{1}(\theta)$ of the envelope $e_{R_{B, f_{c}}}(\theta)$ of the normalized ACR $R_{B, f_{c}}(\theta)$.

3) End-Ambiguity and Asymptotic Thresholds for Passband Pulses: Let us prove that the asymptotic threshold in (37) and the end-ambiguity threshold in (38) of the pulse $w_{B, f_{c}}(t)$ in (42) 


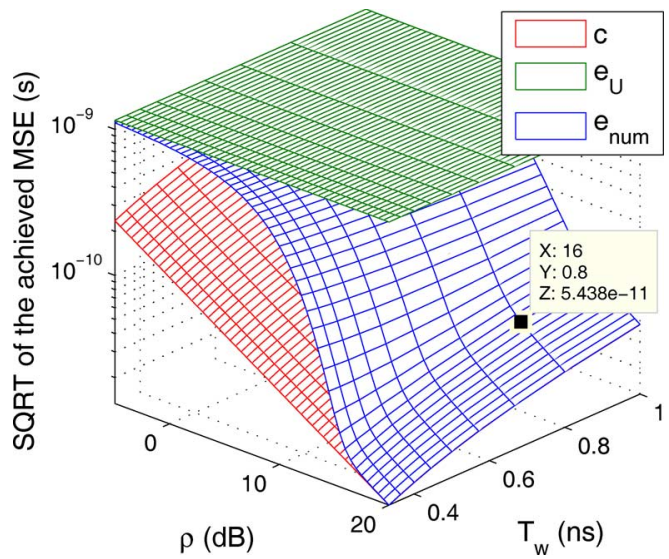

Fig. 1. Baseband: SQRTs of the CRLB $c$, the maximum MSE $e_{U}$ and the MSEA $e_{\text {num }}$ w.r.t. the SNR $\rho$ and the pulse width $T_{w}$.

are function of the shape $w_{1}(t)$ of the envelope $w_{B}(t)$ in (41) and the IFBW $\lambda$ in (15) only.

As $\Delta \approx \frac{1}{f_{c}} \approx \frac{2 \pi}{\beta_{s}}$ for oscillating ACRs, we can write $e_{R}(\Delta)$, $H_{a s}$ and $H_{a m 2}^{j_{c}}$ in (37) and (38) using (45) and (46) as:

$$
\begin{aligned}
e_{R}(\Delta) & =R_{B}\left(\frac{1}{f_{c}}\right)=R_{1}\left(\frac{1}{\lambda}\right) \\
H_{a s} & =-\frac{\left(\alpha_{a s}-1\right)^{2}\left[1-R_{1}\left(\frac{1}{\lambda}\right)\right]}{128 \pi^{3}} \\
H_{a m 2} & =-\frac{\pi \alpha_{a m 2}^{2} \lambda^{4}\left[1-R_{1}\left(\frac{1}{\lambda}\right)\right]}{8 \beta_{1}^{4}} .
\end{aligned}
$$

Hence, the end-ambiguity and asymptotic thresholds of $w_{B, f_{c}}(t)$ are independent of $B$ and $f_{c}$ separately; they depend on the shape $R_{1}(\theta)$ of the envelope of the ACR and on the IFBW $\lambda$. Note that $R_{1}(\theta)$ and $\lambda$ determine together the shape of the ACR of $w_{B, f_{c}}(t)$.

We have mentioned in the introduction that a closed-form expression of the asymptotic threshold is derived in [14] based on the MIE-based MSEA in [16]. The obtained result is very nice. However, it is only applicable on cardinal sine ACRs. Furthermore, the employed MSEA considers the unknown parameter and the zeros of the ACR as testpoints. This choice is not optimal for asymptotic threshold computation because the MSE starts to deviate from the asymptotic MSE (the CRLB for asymptotically estimators) when the estimate starts to fall around the strongest local maxima.

The latter problem is bypassed in [15] by only considering the unknown parameter and the two strongest local maxima (like in our approach). However, the threshold is not computed based on the achieved MSE w.r.t. the asymptotic one (like in the approach of [14] and ours) but based on the probability of non-ambiguity. Obviously, the MSE-based approach is more reliable because the main concern in estimation is to minimize the MSE (by making it attaining the asymptotic one).

As mentioned in the introduction, we have two main contributions with regards to the thresholds. The first contribution is that we derived closed-from expressions of the begin-ambiguity, end-ambiguity and asymptotic thresholds for oscillating and non-oscillating ACRs. The obtained thresholds are very accurate (especially for the end-ambiguity and asymptotic thresholds of oscillating ACRs, see Section V). Our approach can be applied on any estimation problem satisfying the system model

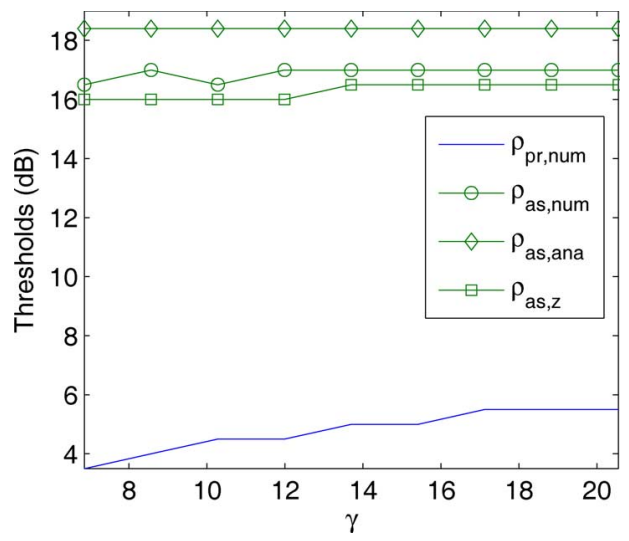

Fig. 2. Baseband: a priori and asymptotic thresholds w.r.t. the ATBW $\gamma$.

of Part I. To the best of our knowledge, our results are completely new. Also, we have dealt with the case of non-oscillating ACRs. To the best of our knowledge, no one has investigated this case before.

The second contribution is that we proved some properties of the obtained thresholds. The proved properties are valid for any estimation problem whose ACR (rather than transmitted signal like in the TOA case) satisfies (41) and (42).

\section{Numerical Results About Thresholds}

In this section we discuss some numerical results about the thresholds obtained for the baseband and passband Gaussian pulses respectively given by

$$
\begin{aligned}
g_{T_{w}}(t) & =e^{-2 \pi \frac{t^{2}}{T_{w}^{2}}} \\
g_{T_{w}, f_{c}}(t) & =e_{s}(t) \cos \left(2 \pi f_{c} t\right) .
\end{aligned}
$$

The bandwidth at $-10 \mathrm{~dB}$ of both $g_{T_{w}}(t)$ and $g_{T_{w}, f_{c}}(t)$ and the MQBW of $g_{T_{w}}(t)$ (equal to the EMQBW of $g_{T_{w}, f_{c}}(t)$ ) can respectively be expressed as [32]:

$$
\begin{aligned}
B & =2 \sqrt{\frac{\ln 10}{\pi}} \frac{1}{T_{w}} \\
\beta^{2} & =\frac{2 \pi}{T_{w}^{2}} .
\end{aligned}
$$

In Sections V-A and V-B we consider the baseband and passband cases, respectively.

\section{A. Baseband Pulses: A Priori and Asymptotic Thresholds w.r.t. the $A T B W$}

We consider in this subsection the baseband pulse in (47) and compute the a priori and asymptotic thresholds w.r.t. the ATBW $\gamma$ in (14) by considering a variable pulse width $T_{w}$ and a fixed a priori domain $D_{\Theta}=[-2,2] \mathrm{ns}$.

In Fig. 1, we show the SQRTs of the CRLB $c$ in (1), the maximum MSE $e_{U}$ in (3), and the MSEA $e_{\text {num }}$ in (6) w.r.t. $\rho$ and $T_{w}$. We can see that $e_{\text {num }}$ decreases as $T_{w}$ decreases for $\rho \geq 16 \mathrm{~dB}$ whereas it becomes approximately constant w.r.t. $T_{w}$ for $\rho<16 \mathrm{~dB}$. In fact, $c$ is achieved at $\rho=16 \mathrm{~dB}$ (approximately equal to the asymptotic threshold), and it is also inversely proportional to $\beta_{s}^{2}$ which is in turn inversely proportional to $T_{w}^{2}$ as can be noticed from (1) and (50). We deduce that 


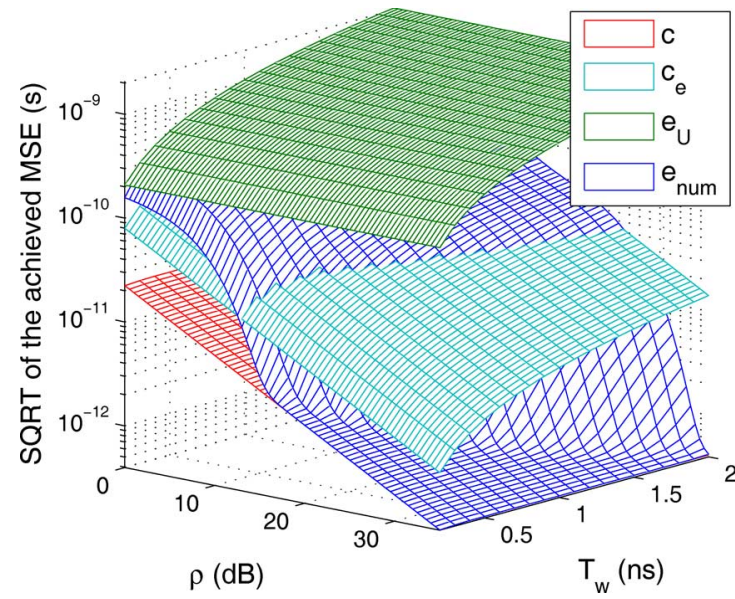

Fig. 3. Passband: SQRTs of the CRLB $c$, the ECRLB $c_{\epsilon}$, the maximum MSE $e_{U}$, and the MSEA $e_{\text {num }}$ w.r.t. the $\operatorname{SNR} \rho$ and the pulse width $T_{w}$.

the MSE can (resp. cannot) be reduced with baseband pulses by increasing the bandwidth (inversely proportional to the pulse width) if the available SNR is above (resp. below) the asymptotic threshold.

Fig. 2 shows the a priori threshold $\rho_{p r, \text { num }}$ (obtained numerically from $e_{\text {num }}$ ), the asymptotic thresholds $\rho_{a s, \text { num }}$ and $\rho_{a s, z}$ (resp. obtained numerically from $e_{\text {num }}$ and the ALB $z_{1}$ in (8)) and the asymptotic threshold $\rho_{a s, \text { ana }}$ in (23) (analytic expression) w.r.t. the ATBW $\gamma$. We can see that:

- The asymptotic thresholds $\rho_{a s, \text { num }}, \rho_{a s, z}$ and $\rho_{a s, \text { ana }}$ are approximately constant $\left(\rho_{a s, \text { num }} \approx 17 \mathrm{~dB}\right.$, $\rho_{a s, z} \approx 16.5 \mathrm{~dB}$ and $\left.\rho_{a s, \text { ana }}=18.5 \mathrm{~dB}\right)$. This result is already proved in Section IV-C.

- The a priori threshold $\rho_{p r \text {;um }}$ increases with $\gamma$; in fact, the gap between the CRLB and the maximum MSE increases with $\gamma$ while the asymptotic threshold is constant.

\section{B. Passband Pulses: A Priori, Begin-Ambiguity, End-Ambiguity and Asymptotic Thresholds Width Respect to the IFBW}

In this subsection we consider the passband pulse in (48). We compute the a priori, begin-ambiguity, end-ambiguity and asymptotic thresholds w.r.t. the IFBW $\lambda$ in (15) by considering variable pulse width $T_{w}$ and a priori domain $D_{\Theta}=[-2,1.5] T_{w}$ and a fixed carrier $f_{c}=6.85 \mathrm{GHz}$.

In Fig. 3, we show the SQRTs of the CRLB $c$ in (1), the ECRLB $c_{e}$ in (2), the maximum MSE $e_{U}$ in (3), and the MSEA $e_{\text {num }}$ in (6) w.r.t. $\rho$ and $T_{w}$. The ambiguity region is not observable for small $T_{w}$ because $e_{\text {num }}$ converges from $e_{U}$ to $c$ without staying long equal to $c_{e}$ due to the weak oscillations in the ACR; this explains why the begin-ambiguity and end-ambiguity thresholds are very close to each other for small $\lambda$ as can be seen in Fig. 4. For high $T_{w}$, the ambiguity region is easily observable; it has a triangular shape due to the gap between the begin-ambiguity and end-ambiguity thresholds that increases with $\lambda$ as can be seen in Fig. 4.

In Fig. 4, we show the a priori threshold $\rho_{p r, \text { num }}$ (obtained numerically from $e_{\text {num }}$ ), begin-ambiguity threshold $\rho_{a m 1, \text { num }}$ (obtained numerically from $e_{\text {num }}$ ), begin-ambiguity threshold $\rho_{a m 1, \text { ana }}$ in (36) (analytic expression), end-ambiguity threshold $\rho_{a m 2, \text { num }}$ (obtained numerically from $e_{\text {num }}$ ), end-ambiguity

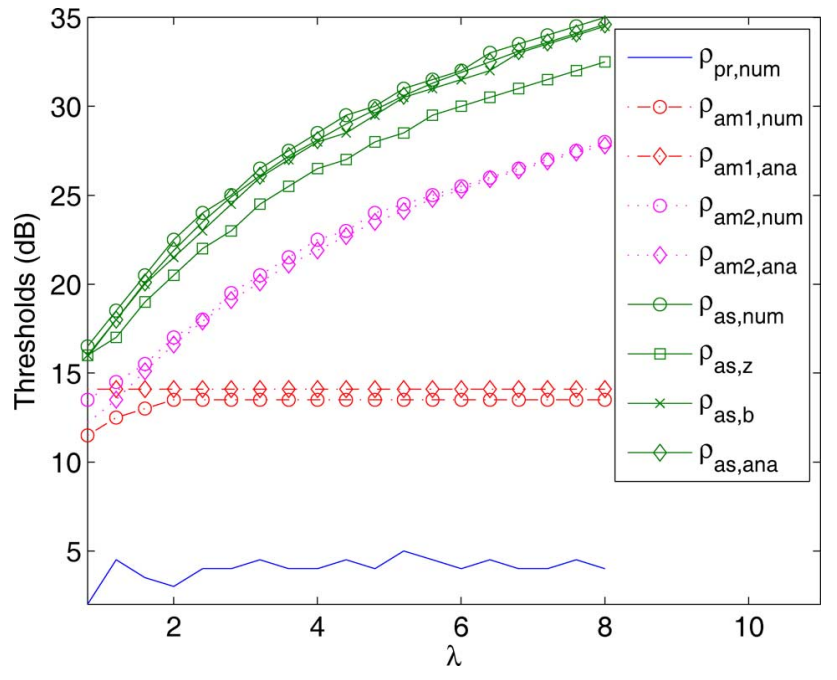

Fig. 4. Passband: A priori, begin-ambiguity, end-ambiguity, and asymptotic thresholds w.r.t. the IFBW $\lambda$.

threshold $\rho_{a m 2, \text { ana }}$ in (38) (analytic expression), asymptotic thresholds $\rho_{a s, \text { num }}, \rho_{a s, z}$ and $\rho_{a s, b}$ (resp. obtained numerically from $e_{\text {num }}$ and the ALBs $z_{1}$ in (8) and $b_{1}$ in (9)) and the asymptotic threshold $\rho_{a s, \text { ana }}$ in (37) (analytic expression) w.r.t. the IFBW $\lambda$. We can see that:

- Both $\rho_{p r, \text { num }}$ and $\rho_{a m 1 . n u m}$ are approximately constant. In fact, the a priori and begin-ambiguity thresholds of a passband signal are approximately equal to the a priori and asymptotic thresholds of its envelope (see Part I). Furthermore, the a priori threshold of the envelope increases with the ATBW (constant here), and its asymptotic threshold is constant (see Section V-A).

- Both $\rho_{a m 2, \text { num }}$ and $\rho_{a s, \text { num }}$ increase with $\lambda$. In fact, the gap between the global and the local maxima of the ACR decreases as $\lambda$ increases. Therefore, a higher SNR is required to guarantee that the estimate will only fall around the global maximum.

- The asymptotic threshold $\rho_{a s, b}$ obtained from the ALB $b_{1}$ is very close to $\rho_{a s, \text { num }}$ whereas $\rho_{a s, z}$ obtained from $z_{1}$ is a bit far from $\rho_{a s, \text { num }}$.

- The thresholds $\rho_{a m 1, \text { ana }}, \rho_{a m 2, \text { ana }}$ and $\rho_{a s, \text { ana }}$ obtained from the analytic expressions are very close to $\rho_{a m 1, \text { num }}$, $\rho_{a m 2, \text { num }}$ and $\rho_{a s, \text { num }}$ obtained numerically. This result validates the accurateness of the analytic thresholds especially because they are obtained by considering one arbitrary envelope and by varying $f_{c}$ according to $\lambda$ whereas the numerical ones are obtained by varying the envelope and fixing $f_{c}$.

Thanks to Fig. 4, we can predict the value of the achievable MSE based on the values of the available SNR and IFBW. It is approximately equal to the maximum $\operatorname{MSE}$ if $(\rho, \lambda)$ falls in the a priori region (below the a priori threshold curve), between the maximum MSE and the ECRLB if $(\rho, \lambda)$ falls in the a priori ambiguity transition region (between the a priori and begin-ambiguity threshold curves), approximately equal to the ECRLB if $(\rho, \lambda)$ falls in the ambiguity region (between the begin-ambiguity and end-ambiguity threshold curves), between the ECRLB and the CRLB if $(\rho, \lambda)$ falls in the ambiguity asymptotic transition region (between the end-ambiguity and asymptotic threshold curves), and approximately equal to 
CRLB if $(\rho, \lambda)$ falls in the asymptotic region (above the asymptotic threshold curve).

To summarize we can say that the $a$ priori threshold depends on both the shape of the envelope of the ACR and the a priori domain. The begin-ambiguity threshold depends only on the shape of the envelope of the ACR function. The end-ambiguity and asymptotic thresholds only depend on the shape of the ACR, or on any set of parameters describing this shape like the shape of the envelope and the IFBW together.

\section{Pulse Design for Minimum AChievable MSE}

We have seen in Sections IV and V that the achievable MSE depends on the available SNR and on the parameters of the transmitted signal. In this section we consider the design of the transmitted pulse spectrum w.r.t. the available SNR $\rho_{0}$ in order to minimize the achievable MSE.

We assume that the transmitted signal consists of the passband Gaussian pulse in (48). Our goal is to find the optimal values $B_{0}$ and $f_{c, 0}$ of the bandwidth $B$ and the carrier frequency $f_{c}$, respectively; the optimal pulse width $T_{w, 0}$ can be obtained from the optimal bandwidth $B_{0}$ using (49).

Regarding the constraints about the spectrum of the transmitted pulse, the two following scenarios are investigated:

i) The spectrum falls in a given frequency band.

ii) The spectrum falls in a given frequency band and has a fixed bandwidth.

To perform the optimization w.r.t. the SNR, the receiver should measure the SNR and feed it back to the transmitter, unless the latter can measure it by itself (such as with mono-static radars). As the SNR should be accurately estimated we investigate in Section VI-A the estimation of the SNR then we treat in Sections VI-B and VI-C the optimization of the spectrum subject to the two constraints introduced above.

\section{A. MLE of the SNR}

In this subsection we derive the MLE $\hat{\rho}$ of the SNR $\rho$ and approximate its statistics.

For convenience we recall from Section II the following equations: $X_{r, s}(\theta)=\alpha R_{s}(\theta-\Theta)+w(\theta), C_{w}(\theta)=\frac{N_{0}}{2} R_{s}(\theta)$ (with $R_{s}(0)=E_{s}$ ) and $\rho=\frac{\alpha^{2} E_{s}}{N_{0} / 2}$. As well, we rewrite from (2) in Part I [1] the expression of the log-likelihood function as: $\Lambda=-\frac{1}{N_{0}}\left[\alpha^{2} E_{s}-2 \alpha X_{r, s}(\theta)\right]$.

By substituting the gain $\alpha$ in the expression of $\Lambda$ by $\sqrt{\frac{\rho N_{0}}{2 E_{s}}}$ and equating the partial derivatives of $\Lambda$ w.r.t. $\rho$ and $\theta$ to zero we can write the MLE of $\rho$ as:

$$
\hat{\rho}=\frac{2 X_{r, s}^{2}(\hat{\Theta})}{E_{s} N_{0}}
$$

where $\hat{\Theta}$ is the MLE of $\Theta$ given in Section II.

We will see later in Sections VI-B and VI-C that our optimization method is useful when the SNR is superior to the begin-ambiguity threshold. Therefore, the maximum $\left[\hat{\Theta}, X_{r, s}(\hat{\Theta})\right]$ of the CCR $X_{r, s}(\theta)$ will fall around the maximum $\left(\Theta, E_{s}\right)$ of the envelope of the ACR $R_{s}(\theta)$ so $X_{r, s}(\hat{\Theta})$ can be approximated by (Taylor series expansion limited to first order) $X_{r, s}(\hat{\Theta}) \approx$
$E_{s}+w(\Theta)$ where $^{2} w(\Theta) \sim \mathcal{N}\left(0, \frac{E_{s} N_{0}}{2}\right)$. Accordingly, $\hat{\rho}$ can be approximated using (51) by

$$
\hat{\rho} \approx \rho(1+\kappa)^{2}
$$

with $\kappa \sim \mathcal{N}\left(0, \frac{1}{\rho}\right)$. As such, $\hat{\rho}$ asymptotically (due to the approximation) follows a non-central Chi-squared distribution with one degree of freedom and with the mean and the variance respectively given by:

$$
\begin{aligned}
& \mu_{\rho}=\rho+1 \\
& \sigma_{\rho}^{2}=4 \rho+2 \approx 4 \rho .
\end{aligned}
$$

As an illustration, we assume that the estimation error is equal to the standard deviation $\sigma_{\rho} \approx 2 \sqrt{\rho}$. Then for $\rho=20 \mathrm{~dB}$ (resp. $\rho=30 \mathrm{~dB}$ ), the SNR estimate is approximately equal to $100+20=120$ (resp. $\hat{\rho} \approx 1000+2 \sqrt{1000}=1063)$ which corresponds to an error of $10 \log (120)-20=0.79 \mathrm{~dB}$ (resp. $10 \log (1063)-30=0.27 \mathrm{~dB})$ on a logarithmic scale. This shows that the obtained estimator is sufficiently accurate to be used in the optimization problems investigated below.

\section{B. Spectrum Falling in a Given Frequency Band}

We assume in this subsection that the spectrum of the transmitted pulse falls in the frequency band $\left[f_{l}, f_{h}\right]$. This constraint can be written as:

$$
C_{1}:\left\{\begin{array}{l}
f_{c}, B>0 \\
f_{c}-\frac{B}{2} \geq f_{l} \\
f_{c}+\frac{B}{2} \leq f_{h}
\end{array}\right.
$$

We consider the FCC UWB band $^{3}\left[f_{l}, f_{h}\right]=[3.1,10.6] \mathrm{GHz}$ [27] in our numerical example.

We can write our optimization problem as:

$$
\left(B_{0}, f_{c, 0}\right)=\underset{\left(B, f_{c}\right)}{\operatorname{argmin}}\{e\} \text { s.t. } \rho=\rho_{0}, C_{1}
$$

where $e$ denotes the achievable MSE. As depicted in Fig. 5, the feasible region corresponding to the constraint $C_{1}$ in (52) is the triangular region (region with horizontal dashed bars) limited by the lines

$$
\begin{aligned}
L_{0}: B & =0 \\
L_{f_{l}}: f_{c} & =f_{l}+\frac{B}{2} \\
L_{f_{h}}: f_{c} & =f_{h}-\frac{B}{2} .
\end{aligned}
$$

The maximum bandwidth in this feasible region is given by

$$
B_{\max }=f_{h}-f_{l}
$$

and corresponds to the intersection of the lines $L_{f_{l}}$ and $L_{f_{h}}$ :

$$
L_{f_{l}} \cap L_{f_{h}}=\left(f_{h}-f_{l}, \frac{f_{l}+f_{h}}{2}\right) .
$$

\footnotetext{
${ }^{2} \mathcal{N}(m, v)$ stands for the normal distribution of mean $m$ and variance $v$.
}

${ }^{3}$ We have chosen the FCC UWB spectrum because it is possible, thanks to its ultra wide authorized band, to move the pulse spectrum around so that the IFBW be reduced and the asymptotic threshold becomes lower than or equal to the available SNR. 


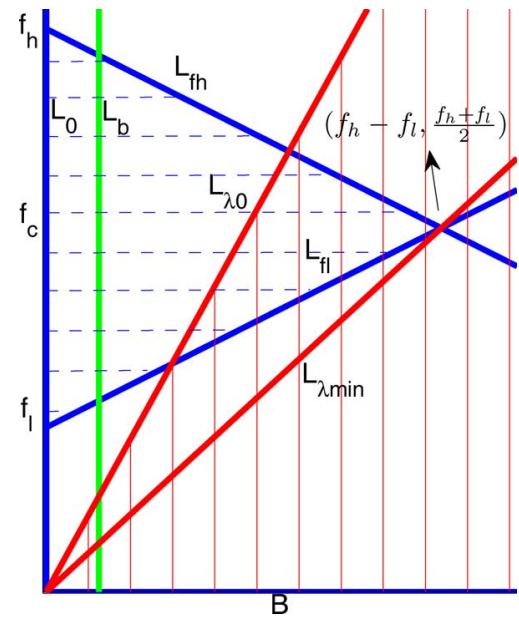

Fig. 5. The feasible regions corresponding to the constraint $C_{1}$ in (52) (region with horizontal dashed bars) and the constraint $C_{2}$ in (65) (region with vertical solid bars).

We have $B_{\max }=7.5 \mathrm{GHz}$ for the FCC UWB band.

For a given bandwidth $B=b$, the minimal and maximal IFBWs in the feasible region of $C_{1}$ are given by

$$
\begin{aligned}
& \lambda_{b, \text { min }}=\frac{f_{l}}{b}+\frac{1}{2} \\
& \lambda_{b, \text { max }}=\frac{f_{h}}{b}-\frac{1}{2}
\end{aligned}
$$

and correspond to the intersections of the line

$$
L_{b}: B=b
$$

with the lines $L_{f_{l}}$ and $L_{f_{h}}$ respectively:

$$
\begin{aligned}
& L_{b} \cap L_{f_{l}}=\left(b, f_{l}+\frac{b}{2}\right) \\
& L_{b} \cap L_{f_{h}}=\left(b, f_{h}-\frac{b}{2}\right) .
\end{aligned}
$$

As result, the minimal IFBW is equal to

$$
\lambda_{\min }=\frac{1}{2}+\frac{f_{l}}{f_{h}-f_{l}}
$$

and corresponds to $L_{f_{l}} \cap L_{f_{h}}$ in (57); we have $\lambda_{\min }=0.913$ for the FCC UWB band. The maximal IFBW is infinite and corresponds to $B=0 \mathrm{GHz}$.

Let us now consider the minimization of the achievable MSE. According to the value of the available SNR $\rho_{0}$, three cases can be considered:

i) The available SNR is lower than the begin-ambiguity threshold: $\rho_{0}<\rho_{a m 1} ; \rho_{a m 1}$ is constant because it depends on the envelope shape only.

ii) The available SNR is close to the begin-ambiguity threshold: $\rho_{0} \approx \rho_{a m 1}$.

iii) The available SNR is greater than the begin-ambiguity threshold: $\rho_{0}>\rho_{a m 1}$.

Consider the first case where $\rho_{0}<\rho_{a m 1}$. We have seen in Part I [1] that a passband signal and its envelope approximately achieve the same MSE below the begin-ambiguity threshold of the passband signal (approximately equal to the asymptotic threshold of the envelope). We have also seen in Section V-A that below the asymptotic threshold of the envelope, the achieved MSE is approximately constant and does not depend on the pulse width and the bandwidth. Therefore, nothing can be done to reduce the MSE in this case.

Consider the second case where $\rho_{0} \approx \rho_{a m 1}$. As the ECRLB $c_{e}$ in (2) is approximately achieved in this case, we minimize the MSE by maximizing the bandwidth $B$ (i.e., minimizing the pulse width $T_{w}$ ) so the EMQBW $\beta_{e}^{2}$ in (2) is maximized and $c_{e}$ (inversely proportional to $\beta_{e}^{2}$ ) is minimized. Therefore, the optimal solution $\left(B_{0}, f_{c, 0}\right)$ in this case and the corresponding achievable MSE $e_{0}$ are given by

$$
\left\{\begin{array}{l}
\left(B_{0}, f_{c, 0}\right)=\left(f_{h}-f_{l}, \frac{f_{l}+f_{h}}{2}\right) \\
e_{0} \approx \frac{1}{\rho_{0} \beta_{e, 0}^{2}}=\frac{T_{w, 0}^{2}}{2 \pi \rho_{0}}=\frac{2 \ln 10}{\pi^{2} B_{0}^{2} \rho_{0}}
\end{array}\right.
$$

where the expression of $e_{0}$ is obtained using (49) and (50). Note that $f_{h}-f_{l}$ is the maximum bandwidth $B_{\max }$ in (56). As $\rho_{a m 1} \approx$ $14 \mathrm{~dB}$ as can be seen in Fig. 4, we have $e_{0} \approx 330.24 \mathrm{ps}^{2}$ for the FCC band $\left(B_{0}=7.5 \mathrm{GHz}\right)$.

Consider now the last case where $\rho_{0}>\rho_{a m 1}$. As we can see in Fig. 4, the point $\left(\rho_{0}, \lambda\right)$ will fall, according to the value of the IFBW $\lambda$, in the ambiguity region, the ambiguity-asymptotic transition region, or the asymptotic region. Therefore, the achievable MSE is equal to the ECRLB $c_{e}$, between the ECRLB and the CRLB $c$, or equal to the CRLB. Now, in order to find the optimal bandwidth $B_{0}$ and carrier frequency $f_{c, 0}$ we proceed as follows:

1) We pick from Fig. 4 the value $\lambda_{0}$ of the IFBW $\lambda$ for which the available SNR $\rho_{0}$ belongs to the asymptotic threshold curve.

2) In order to guarantee that the CRLB is achieved, we consider the constraint that $\lambda$ is lower than or equal to the picked $\lambda_{0}$. If this constraint cannot be satisfied because $\rho_{0}$ is lower than the minimal IFBW $\lambda_{\min }$ in (63), then the CRLB cannot be achieved. In order to make the achievable MSE the closest possible to the CRLB, we set $\lambda$ to the minimal IFBW $\lambda_{\min }$. This constraint can be expressed as

$$
C_{2}: \begin{cases}\lambda=\frac{f_{c}}{B} \leq \lambda_{0} & \text { if } \lambda_{0} \geq \lambda_{\min } \\ \lambda=\frac{f_{c}}{B}=\lambda_{\min } & \text { if } \lambda_{0}<\lambda_{\min }\end{cases}
$$

3) Now, given that the estimator achieves the CRLB or a MSE that is the closest possible to the CRLB thanks to the previous step, we minimize the achievable MSE by minimizing the CRLB itself.

According to the last step, we can write from (52) and (65) the minimization problem in (53) as

$$
\left(B_{0}, f_{c, 0}\right)=\underset{\left(B, f_{c}\right)}{\operatorname{argmin}}\{c\} \quad \text { s.t. } C_{1}, C_{2} .
$$

As $c$ can be approximated from (1) and (4) by

$$
c=\frac{1}{\rho \beta_{s}^{2}}=\frac{1}{\rho\left(\beta_{e}^{2}+4 \pi^{2} f_{c}^{2}\right)} \approx \frac{1}{\rho 4 \pi^{2} f_{c}^{2}}
$$

we can write the minimization problem in (66) as

$$
\left(B_{0}, f_{c, 0}\right)=\underset{\left(B, f_{c}\right)}{\operatorname{argmax}}\left\{f_{c}\right\} \text { s.t. } C_{1}, C_{2} .
$$

As shown in Fig. 5, the feasible region of the constraint $C_{2}$ in (65) is the half-space below the line $L_{\lambda_{0}}: f_{c}=\lambda_{0} B$ (region with vertical solid bars). We have already seen that the feasible region of the constraint $C_{1}$ in (52) is the triangle limited by the lines $L_{0}, L_{f_{l}}$ and $L_{f_{h}}$. Therefore, the feasible region of $C_{1}$ and 


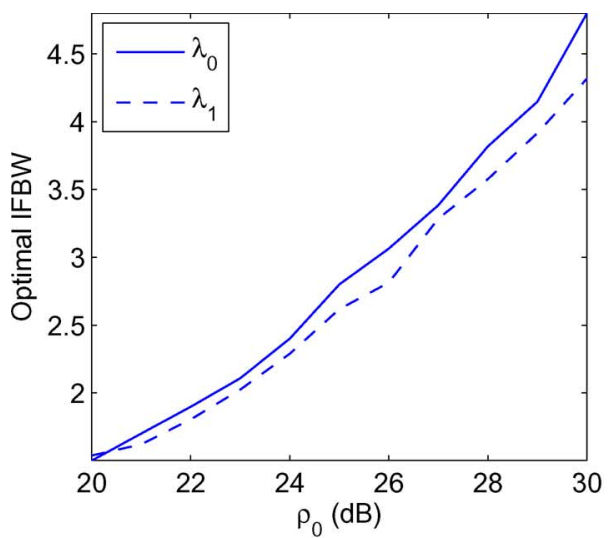

(a)

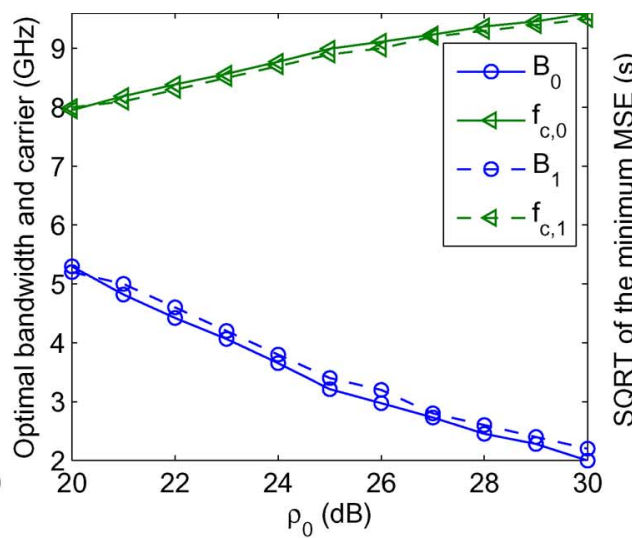

(b)

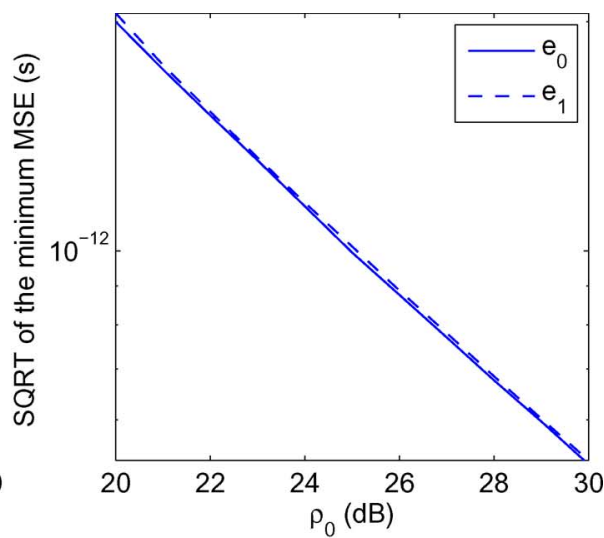

(c)

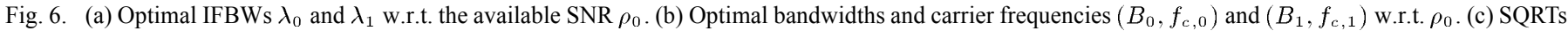
of the optimal MSEs $e_{0}$ and $e_{1}$ w.r.t. $\rho_{0}\left(\lambda_{1}, B_{1}, f_{c, 1}\right.$ and $e_{1}$ are obtained from exhaustive search based on $\left.e_{\text {num }}\right)$.

$C_{2}$ together is the triangular region limited by $L_{f_{l}}, L_{f_{h}}$ and $L_{\lambda_{0}}$ (region with both vertical and horizontal bars). Consequently, the solution of the maximization problem in (68) corresponds to the point of intersection $\left(\frac{2}{2 \lambda_{0}+1} f_{h}, \frac{2 \lambda_{0}}{2 \lambda_{0}+1} f_{h}\right)$ of the lines $L_{f_{h}}$ and $L_{\lambda_{0}}$ as can easily be seen in Fig. 5 . In the special case where $\lambda_{0}<\lambda_{\min }$, the feasible region of $C_{2}$ reduces to the line $L_{\lambda_{\text {min }}}: f_{c}=\lambda_{\min } B$ so the feasible region of $C_{1}$ and $C_{2}$ reduces to the point $\left(f_{h}-f_{l}, \frac{f_{l}+f_{h}}{2}\right)$ which is as result the solution of (68).

Finally, the solution when the available SNR is larger than the begin-ambiguity threshold and the corresponding achievable MSE are given by:

$$
\begin{aligned}
& \left\{\begin{array}{l}
\left(B_{0}, f_{c, 0}\right)=\left(f_{h}-f_{l}, \frac{f_{l}+f_{h}}{2}\right) \\
\left.e_{0} \in\right] \frac{1}{4 \pi^{2} f_{c, 0}^{2} \rho_{0}}, \frac{2 \ln 10}{\pi^{2} B_{0}^{2} \rho_{0}}[
\end{array} \text { if } \quad \lambda_{0}<\lambda_{\min }\right. \\
& \left\{\begin{array}{l}
\left(B_{0}, f_{c, 0}\right)=\left(\frac{2 f_{h}}{2 \lambda_{0}+1}, \frac{2 \lambda_{0} f_{h}}{2 \lambda_{0}+1}\right) \\
e_{0}=\frac{1}{4 \pi^{2} f_{c, 0}^{2} \rho_{0}}
\end{array} \quad \text { if } \quad \lambda_{0} \geq \lambda_{\text {min }}\right.
\end{aligned}
$$

with $\frac{1}{4 \pi^{2} f_{c, 0}^{2} \rho_{0}}$ being the CRLB at the SNR $\rho_{0}$, and $\frac{2 \ln 10}{\pi^{2} B_{0}^{2} \rho_{0}}$ the minimum MSE in (64) achieved when $\rho_{0} \approx \rho_{a m 1}$.

Note that in practice we do not need to compute the optimal bandwidth and carrier frequency in real time; it suffices to calculate them once w.r.t. the SNR and to save the obtained values in a table. Then during the communication the receiver measures the SNR and feeds it back to the transmitter which, in turn, selects the optimal bandwidth and carrier frequency from the table and tunes the spectrum of the transmitted signal to meet the optimal one.

Let us now discuss a numerical example about the scenario considered in this subsection. We denote by $\left(B_{1}, f_{c, 1}\right)$ the point minimizing the MSEA $e_{\text {num }}$ in the band $\left[f_{l}, f_{h}\right]=[3.1,10.6] \mathrm{GHz}, e_{1}$ the minimal $e_{\mathrm{num}}$, and $\lambda_{1}$ the corresponding IFBW. To obtain $\left(B_{1}, f_{c, 1}\right), e_{1}$ and $\lambda_{1}$, the available band is swept (exhaustive search) using an increment of $0.2 \mathrm{GHz}$ for the bandwidth $B$ and $0.1 \mathrm{GHz}$ for the carrier frequency $f_{c}$.

In Fig. 6(a) we show $\lambda_{0}$ (obtained from our method) and $\lambda_{1}$, both w.r.t. the available $\operatorname{SNR} \rho_{0}$. We can see that $\lambda_{1}$ is a bit smaller than $\lambda_{0}$. This is due to the factor $\alpha_{a s}=1.1$ in the definition of the asymptotic threshold in (13). For $\rho_{0}=22 \mathrm{~dB}$, we have $\lambda_{0}=1.9$ and $\lambda_{1}=1.8$.

In Fig. 6(b) we show $B_{0}$ and $f_{c, 0}$ (obtained from our method), and $B_{1}$ and $f_{c, 1}$ w.r.t. $\rho_{0}$. We can see that $B_{0}$ and $f_{c, 0}$ are very close to $B_{1}$ and $f_{c, 1}$, respectively. This result shows that our solution is very close to the optimal one. We can also see that $B_{1}$ (resp. $f_{c, 1}$ ) is a bit larger (resp. lower) than $B_{0}$ (resp. $\left.f_{c, 0}\right)$. In fact, $\lambda_{1} \lesssim \lambda_{0}$ as already observed in Fig. 6(a). For $\rho_{0}=22 \mathrm{~dB}$, we have $\left(B_{0}, f_{c, 0}\right)=(4.42,8.39) \mathrm{GHz}$ and $\left(B_{1}, f_{c, 1}\right)=(4.6,8.3) \mathrm{GHz}$.

In Fig. 6(c) we show the SQRTs of $e_{0}$ (minimum MSE obtained from our method) and $e_{1}$ w.r.t. $\rho_{0}$. We can see that $e_{0}$ and $e_{1}$ are very close to each other. For $\rho_{0}=22 \mathrm{~dB}$, we have $e_{0}=2.27 \mathrm{ps}^{2}$ and $e_{1}=2.32 \mathrm{ps}^{2}$.

\section{Spectrum Falling in a Given Frequency Band and Having a Fixed Bandwidth}

We assume here that the spectrum of the transmitted pulse falls in the frequency band $\left[f_{l}, f_{h}\right]$ and has the fixed bandwidth $B=b$. The constraint about the bandwidth can be written as:

$$
C_{3}: B=b
$$

The feasible region corresponding to the constraints $C_{1}$ in (52) and $C_{3}$ in (69) is the segment of the line $L_{b}$ in (60) limited by the lines $L_{f_{l}}$ in (54) and $L_{f_{h}}$ in (55); in this feasible region, the IFBW satisfies:

$$
\lambda \in\left[\lambda_{b, \min }, \lambda_{b, \max }\right]
$$

where $\lambda_{b, \min }$ is given in (58) and $\lambda_{b, \max }$ in (59).

To minimize the MSE, the available $\operatorname{SNR} \rho_{0}$ should fall in the asymptotic region; accordingly, we write the following constraint similarly to the constraint $C_{2}$ in (65):

$$
C_{4}: \begin{cases}\lambda=\frac{f_{c}}{B}=\lambda_{b, \min } & \text { if } \lambda_{0}<\lambda_{b, \min } \\ \lambda=\frac{f_{c}}{B} \leq \lambda_{0} & \text { if } \lambda_{b, \min } \leq \lambda_{0} \leq \lambda_{b, \max } \\ \lambda=\frac{f_{c}}{B}=\lambda_{b, \max } & \text { if } \lambda_{0}>\lambda_{b, \max }\end{cases}
$$




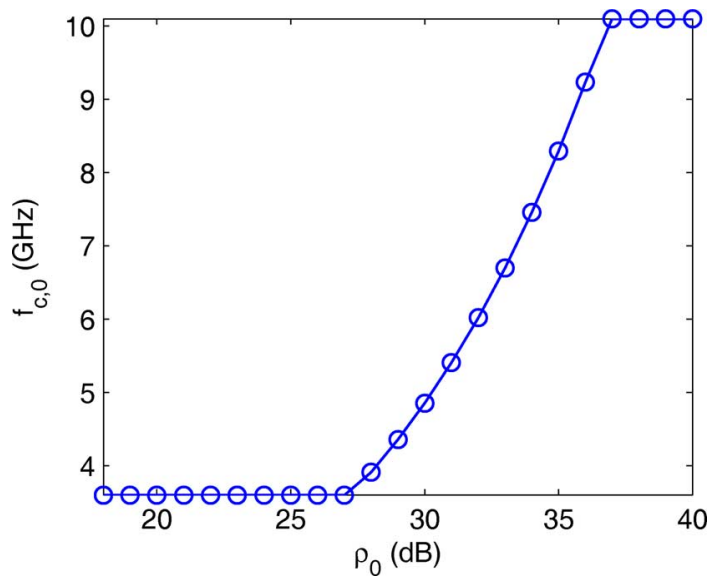

Fig. 7. Optimal carrier frequency $f_{c, 0}$ w.r.t. the available $\operatorname{SNR} \rho_{0}$ for $b=$ $1 \mathrm{GHz}$ (bandwidth).

Our optimization problem can be formulated as:

$$
f_{c, 0}=\underset{f_{c}}{\operatorname{argmax}}\left\{f_{c}\right\} \text { s.t. } C_{1}, C_{3}, C_{4} .
$$

The solution of (71) is $L_{b} \cap L_{f_{l}}$ in (61) for $\lambda_{0}<\lambda_{b, \min }, L_{b} \cap L_{f_{h}}$ in (62) for $\lambda_{0}>\lambda_{b, \max }$, and

$$
L_{b} \cap L_{\lambda_{0}}=\left(b, \lambda_{0} b\right)
$$

for $\lambda_{b, \min } \leq \lambda_{0} \leq \lambda_{b, \max }$.

We can write the solution of our optimization problem and the corresponding achievable MSE as:

$$
\begin{aligned}
& \left\{\begin{array}{l}
f_{c, 0}=f_{l}+\frac{b}{2} \\
e_{0} \in I_{e_{0}}
\end{array} \text { if } \quad \lambda_{0}<\lambda_{b, \min }\right. \\
& \left\{\begin{array}{l}
f_{c, 0}=\lambda_{0} b \\
e_{0}=\frac{1}{4 \pi^{2} f_{c, 0}^{2} \rho_{0}}
\end{array} \text { if } \quad \lambda_{b, \min } \leq \lambda_{0} \leq \lambda_{b, \max }\right. \\
& \left\{\begin{array}{l}
f_{c, 0}=f_{h}-\frac{b}{2} \\
e_{0}=\frac{1}{4 \pi^{2} f_{c, 0}^{2} \rho_{0}}
\end{array} \quad \text { if } \quad \lambda_{0}>\lambda_{b, \max }\right.
\end{aligned}
$$

with $\left.I_{e_{0}}=\right] \frac{1}{4 \pi^{2} f_{c, 0}^{2} \rho_{0}}, \frac{2 \ln 10}{\pi^{2} B_{0}^{2} \rho_{0}}[$.

To point out the improvement that our method brought about, we consider the following numerical example. In Fig. 7 we show the optimal carrier frequency $f_{c, 0}$ w.r.t. the available SNR $\rho_{0}$ for a bandwidth of $B=b=1 \mathrm{GHz}$. The curve of $f_{c, 0}$ has three branches corresponding to the three cases in (73):

- Branch $1([18,27] \mathrm{dB})$ : where it is impossible to get to the asymptotic region because $f_{c, 0}$ cannot decrease more due to $C_{1}$ and $C_{3}$.

- Branch $2([27,37] \mathrm{dB})$ : where the asymptotic region is attained as well as $f_{c, 0}$ increases with $\rho_{0}$.

- Branch $3([37,40] \mathrm{dB})$ : where the asymptotic region is attained but $f_{c, 0}$ cannot increase more due to $C_{1}$ and $C_{3}$.

In Fig. 8 we show the SQRTs of the MSEs $e_{0}, e_{h}$ and $e_{l}$ achieved by the optimal carrier frequency $f_{c .0}$, the carrier frequency $f_{c, h}=f_{h}-\frac{b}{2}$ minimizing the CRLB (without taking account of the ambiguity effect) and the carrier frequency $f_{c, l}=$ $f_{l}+\frac{b}{2}$ minimizing the IFBW ( $f_{c, h}$ and $f_{c, l}$ are constant). To compute the achieved MSEs we first evaluate the IFBW for each of $f_{c, 0}, f_{c . h}$ and $f_{c, l}$ w.r.t. $\rho_{0}$. Then, the MSE is approximated by the ECRLB in (2) if $\rho_{0}$ is between the begin-ambiguity and end-ambiguity thresholds (which are functions of the IFBW)

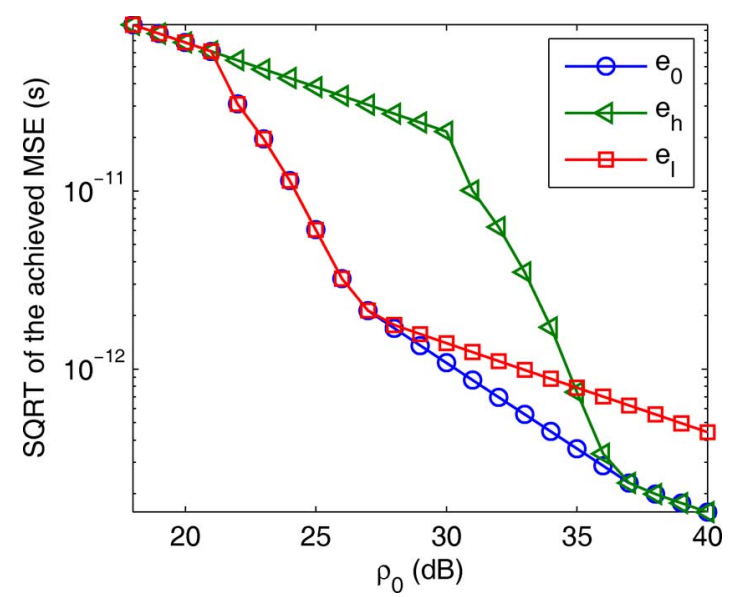

Fig. 8. SQRTs of the MSEs $e_{0}, e_{h}$ and $e_{l}$ achieved by $f_{c, 0}, f_{c, h}$ and $f_{c, l}$ w.r.t. the available SNR $\rho_{0}$ for $b=1 \mathrm{GHz}$ (bandwidth).

and by the MSEA in (7) if $\rho_{0}$ is larger than the end-ambiguity threshold. We can see that $e_{0}$ has four branches:

- Branch $1([18,21] \mathrm{dB})$ : where the ECRLB is achieved; $e_{0}$ decreases only thanks to $\rho_{0}$ because $b$ is constant.

- Branch $2([21,27] \mathrm{dB})$ : where $e_{0}$ converges from the ECRLB to the CRLB.

- Branch $3([27,37] \mathrm{dB})$ : where $e_{0}$ attains the CRLB which decreases thanks to both $\rho_{0}$ and $f_{c, 0}$ (note that $f_{c, 0}$ increases with $\rho_{0}$, see Fig. 7).

- Branch $4([37,40] \mathrm{dB})$ : where $e_{0}$ attains the CRLB which decreases only thanks to $\rho_{0}$ because $f_{c, 0}$ is constant.

Each of $e_{h}$ and $e_{l}$ has only three branches (similar to Branches 1,2 and 4 of $\left.e_{0}\right) ; e_{0}$ highly outperforms $e_{h}\left(e_{h}=19.9^{2} e_{0}\right.$ at $30 \mathrm{~dB}$ ) because $f_{c, 0}$ simultaneously achieves and minimizes the CRLB whereas $f_{c, h}$ just minimizes the CRLB; $e_{0}$ outperforms $e_{l}$ because $f_{c, l}$ achieves the CRLB without minimizing it. The maximum improvement due to the minimization of the CRLB is given by $\left(\frac{f_{c, h}}{f_{c, l}}\right)^{2}<\left(\frac{f_{h}}{f_{l}}\right)^{2}=3.4^{2}$. Fig. 8 shows that $\frac{e_{l}}{e_{0}}=$ $\left(\frac{f_{c, h}}{f_{c, l}}\right)^{2}=2.8^{2}$ for $\rho_{0} \geq 37 \mathrm{~dB}$.

In Sections VI-B and VI-C we have considered two typical optimization examples. More setups with other pulse shapes and with other constraints can be investigated. For TOA estimation based on modulated pulses we exactly follow the procedure described above regardless of the shape of the envelope. The solution of any optimization problem suffering from threshold and ambiguity effects consists in general in the following two steps:

1) Define w.r.t. the tunable parameters (both the carrier frequency and the bandwidth for the problem in Section VI-B and just the carrier frequency for the problem in Section VI-C) the feasible region where the CRLB is achieved.

2) Minimize the CRLB in the feasible region by taking into account the different constraints.

We have mentioned in the introduction that optimal timebandwidth product design is considered in [19] based on the MIE; the mentioned work is based on the probability of non-ambiguity rather than the MSE. Therefore, the obtained solution is optimal for sufficiently high SNRs only.

As mentioned in the introduction, we have one main contribution with regards to optimization subject to threshold and am- 
biguity phenomena. We considered the problem of pulse design for TOA estimation and proposed a very simple algorithm that minimizes the achievable MSE. To the best of our knowledge, this work has never been done before. The obtained solution is completely different from the one obtained by minimizing the CRLB (e.g, [17]). When the threshold and ambiguity phenomena are not taken into account, then the optimal solution consists in filling the available spectrum with the maximum allowed PSD starting from the highest frequency. The works in [17], [19] correspond to the second step of our optimization method.

Finally, we would like to point out that the results of Section VI can be used in practical UWB-based positioning systems where both the multipath component (MPC) resolvability and the perfect multiuser interference suppression can be insured. In fact, TOA estimation can achieve, in multipath line-of-sight (LOS) channels, the same performance as in AWGN channels if the MPCs are resolvable (e.g, see [33] where the CRLB is experimentally attained).

\section{CONCLUSION}

We have employed the MIE-based MSEA to derive analytic expressions for the begin-ambiguity, end-ambiguity and asymptotic thresholds. The obtained thresholds are very accurate, and also can be used with various estimation problems. We have proved that the begin-ambiguity threshold only depends on the shape of the ACR envelope, and the end-ambiguity and asymptotic thresholds only on the shape of the ACR. Therefore, the asymptotic threshold is constant for baseband pulses with a given shape (e.g, Gaussian, cardinal sine, raised cosine with constant roll-off). For passband pulses with given envelope shape, the begin-ambiguity threshold is constant whereas the end-ambiguity and asymptotic thresholds are functions of the IFBW. We have exploited the information on the begin-ambiguity and asymptotic thresholds to optimize, according to the available SNR, the pulse spectrum that achieves the minimum attainable MSE. The proposed method is very simple and very accurate.

\section{REFERENCES}

[1] A. Mallat, S. Gezici, D. Dardari, C. Craeye, and L. Vandendorpe, "Statistics of the MLE and approximate upper and lower bounds-Part I: Application to TOA estimation," IEEE Trans. Signal Process., vol. 62, no. 21, pp. 5663-5676, 2014.

[2] J. Ziv and M. Zakai, "Some lower bounds on signal parameter estimation," IEEE Trans. Inf. Theory, vol. 15, no. 3, pp. 386-391, May 1969.

[3] S.-K. Chow and P. Schultheiss, "Delay estimation using narrow-band processes," IEEE Trans. Acoust., Speech, Signal Process., vol. 29, no. 3, pp. 478-484, Jun. 1981.

[4] A. Weiss and E. Weinstein, "Fundamental limitations in passive time delay estimation-Part I: Narrow-band systems," IEEE Trans. Acoust., Speech, Signal Process., vol. 31, no. 2, pp. 472-486, Apr. 1983.

[5] E. Weinstein and A. Weiss, "Fundamental limitations in passive timedelay estimation-Part II: Wide-band systems," IEEE Trans. Acoust., Speech, Signal Process., vol. 32, no. 5, pp. 1064-1078, Oct. 1984.

[6] A. Zeira and P. Schultheiss, "Realizable lower bounds for time delay estimation," IEEE Trans. Signal Process., vol. 41, no. 11, pp. 3102-3113, Nov. 1993

[7] A. Zeira and P. Schultheiss, "Realizable lower bounds for time delay estimation. 2. Threshold phenomena," IEEE Trans. Signal Process., vol. 42, no. 5, pp. 1001-1007, May 1994.
[8] B. Sadler and R. Kozick, "A survey of time delay estimation performance bounds," in Proc. 4th IEEE Workshop Sensor Array, Multichannel Process., Jul. 2006, pp. 282-288.

[9] B. Sadler, L. Huang, and Z. Xu, "Ziv-Zakai time delay estimation bound for ultra-wideband signals," in Proc. IEEE Int. Conf. Acoust., Speech, Signal Process. (ICASSP), Apr. 2007, vol. 3, pp. III-549-III-552.

[10] A. Renaux, P. Forster, E. Chaumette, and P. Larzabal, "On the high-SNR conditional maximum-likelihood estimator full statistical characterization," IEEE Trans. Signal Process., vol. 54, no. 12, pp. 4840-4843, Dec. 2006.

[11] A. Renaux, P. Forster, E. Boyer, and P. Larzabal, "Unconditional maximum likelihood performance at finite number of samples and high signal-to-noise ratio," IEEE Trans. Signal Process., vol. 55, no. 5, pp. 2358-2364, May 2007.

[12] Bayesian Bounds for Parameter Estimation and Nonlinear Filtering/ Tracking, H. L. Van Trees and K. L. Bell, Eds. New York, NY, USA: Wiley-IEEE Press, 2007.

[13] A. Renaux, "Contributionà l'analyse des Performances D'estimation en Traitement Statistique du Signal," Ph.D. dissertation, ENS CACHAN, Cachan, France, 2006.

[14] A. Steinhardt and C. Bretherton, "Thresholds in frequency estimation," in Proc. IEEE Int. Conf. Acoust., Speech, Signal Process. (ICASSP), Apr. 1985, vol. 10, pp. 1273-1276.

[15] C. Richmond, "Capon algorithm mean-squared error threshold SNR prediction and probability of resolution," IEEE Trans. Signal Process., vol. 53, no. 8, pp. 2748-2764, Aug. 2005.

[16] D. Rife and R. Boorstyn, "Single tone parameter estimation from discrete-time observations," IEEE Trans. Inf. Theory, vol. 20, no. 5, pp. 591-598, Sep. 1974.

[17] Y. Karisan, D. Dardari, S. Gezici, A. D’Amico, and U. Mengali, "Range estimation in multicarrier systems in the presence of interference: Performance limits and optimal signal design," IEEE Trans. Wireless Commun., vol. 10, no. 10, pp. 3321-3331, Oct. 2011.

[18] R. McAulay and D. Sakrison, "A PPM/PM hybrid modulation system," IEEE Trans. Commun. Technol., vol. 17, no. 4, pp. 458-469, Aug. 1969.

[19] H. L. Van Trees, Detection, Estimation, and Modulation Theory, Part I. New York, NY, USA: Wiley, 1968.

[20] P. M. Woodward, Probability and Information Theory With Applications To Radar. New York, NY, USA: McGraw-Hill, 1955.

[21] V. A. Kotelnikov, The Theory of Optimum Noise Immunity. New York, NY, USA: McGraw-Hill, 1959.

[22] J. M. Wozencraft and I. M. Jacobs, Principles of Communication Engineering. New York, NY, USA: Wiley, 1965.

[23] E. Boyer, P. Forster, and P. Larzabal, "Nonasymptotic statistical performance of beamforming for deterministic signals," IEEE Signal Process. Lett., vol. 11, no. 1, pp. 20-22, Jan. 2004.

[24] F. Athley, "Threshold region performance of maximum likelihood direction of arrival estimators," IEEE Trans. Signal Process., vol. 53, no. 4, pp. 1359-1373, Apr. 2005.

[25] L. Najjar-Atallah, P. Larzabal, and P. Forster, "Threshold region determination of ML estimation in known phase data-aided frequency synchronization," IEEE Signal Process. Lett., vol. 12, no. 9, pp. 605-608, Sep. 2005.

[26] C. Richmond, "Mean-squared error and threshold SNR prediction of maximum-likelihood signal parameter estimation with estimated colored noise covariances," IEEE Trans. Inf. Theory, vol. 52, no. 5, pp. 2146-2164, May 2006.

[27] Revision of Part 15 of the Commission Rules Regarding Ultra-Wideband Transmission Systems, FCC 02-48, Federal Communications Commission (FCC), Apr. 2002.

[28] A. Genz, "Numerical computation of multivariate normal probabilities," J. Comput. Graph. Statist., vol. 1, no. 2, pp. 141-149, Jun. 1992.

[29] A. Genz, "On a number-theoretical integration method," Aequationes Mathematicae, vol. 8, no. 3, pp. 304-311, Oct. 1972.

[30] A. Genz, "Randomization of number theoretic methods for multiple integration," SIAM J. Numer. Anal., vol. 13, no. 6, pp. 904-914, Dec. 1976.

[31] D. Nuyens and R. Cools, "Fast component-by-component construction, A reprise for different kernels," in Monte-Carlo and Quasi-Monte Carlo Methods, H. Niederreiter and D. Talay, Eds. New York, NY, USA: Springer-Verlag, 2004, pp. 371-385.

[32] D. Dardari, C.-C. Chong, and M. Win, "Threshold-based time-of-arrival estimators in UWB dense multipath channels," IEEE Trans. Commun., vol. 56, no. 8, pp. 1366-1378, Aug. 2008. 


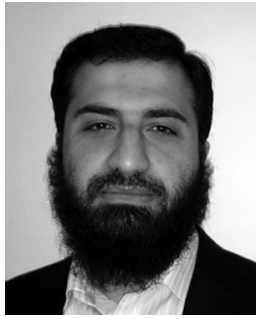

Achraf Mallat (S'06-M'14) received his B.Sc. degree in Electrical and Electronics Engineering from the Lebanese University, Faculty of Engineering, Branch I, Tripoli, Lebanon, in 2002, his M.Sc. degree in Signal, Telecommunications, Image and Radar from the Université de Rennes 1, Rennes, France, in 2004, and his Ph.D. degree in Engineering Sciences from the Université Catholique de Louvain (UCL), Louvain-la-Neuve, Belgium, in 2013. From September 2005 to August 2013, he was a Research/Teaching Assistant at the Louvain School of Engineering, UCL. Since September 2013, he has been a Postdoctoral Researcher with the Institute for Information and Communication Technologies, Electronics and Applied Mathematics, UCL. His current research interests are in the areas of signal processing and parameter estimation, and, in particular, in ultra-wideband (UWB) based positioning and automotive radar systems.

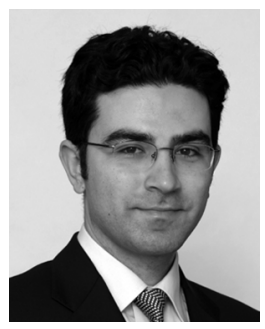

Sinan Gezici (S'03-M'06-SM'11) received the B.S degree from Bilkent University, Turkey, in 2001, and the Ph.D. degree in Electrical Engineering from Princeton University in 2006. From 2006 to 2007, he worked at Mitsubishi Electric Research Laboratories, Cambridge, MA, USA. Since 2007, he has been with the Department of Electrical and Electronics Engineering at Bilkent University, where he is currently an Associate Professor. Dr. Gezici's research interests are in the areas of detection and estimation theory, wireless communications, and localization systems. Among his publications in these areas is the book Ultrawideband Positioning Systems: Theoretical Limits, Ranging Algorithms, and Protocols (Cambridge Univ. Press, 2008). Dr. Gezici is an associate editor for the IEEE TRANSACTIONS ON COMMUNICATIONS, the IEEE WiRELESS COMMUNICATIONS LETTERS, and the Journal of Communications and Networks.

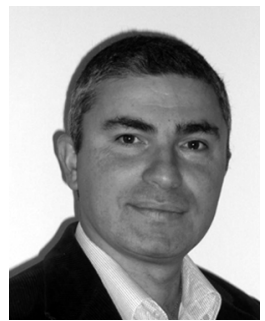

Davide Dardari (M'95-SM'07) received the Laurea degree in electronic engineering (summa cum laude) and the Ph.D. degree in electronic engineering and computer science from the University of Bologna, Italy, in 1993 and 1998, respectively. $\mathrm{He}$ is an Associate Professor at the University of Bologna at Cesena, Italy, where he participates with WiLAB (Wireless Communications Laboratory). Since 2005, he has been a Research Affiliate at Massachusetts Institute of Technology (MIT), Cambridge, USA. He is also Research Affiliate at IEIIT/CNR (National Research Council) and CNIT (Consorzio Nazionale Interuniversitario per le Telecomunicazioni). He published more than 150 technical papers and played several important roles in various National and European Projects. He is co-author of the books Wireless Sensor and Actuator Networks: Enabling Technologies, Information Processing and Protocol Design (Elsevier, 2008) and Satellite and Terrestrial Radio Positioning Techniques-A Signal Processing Perspective (Elsevier, 2011). His interests are on ultra-wide bandwidth (UWB) systems, ranging and localization techniques, distributed signal processing, as well as wireless sensor networks. He received the IEEE Aerospace and Electronic Systems Society's M. Barry Carlton Award (2011) and the IEEE Communications Society Fred W. Ellersick Prize (2012). Prof. Dardari is
Senior Member of the IEEE where he was the Chair for the Radio Communications Committee of the IEEE Communication Society. He was Co-General Chair of the 2011 IEEE International Conference on Ultra-Wideband and co-organizer of the first and second IEEE International Workshop on Advances in Network Localization and Navigation (ANLN) - ICC 2013-2014. He was also Co-Chair of the Wireless Communications Symposium of the 2007 IEEE International Conference on Communications, and Co-Chair of the 2006 IEEE International Conference on Ultra-Wideband. He served as Lead Editor for the EURASIP Journal on Advances in Signal Processing (Special Issue on Cooperative Localization in Wireless Ad Hoc and Sensor Networks), Guest Editor for PROCEEDINGS OF THE IEEE (Special Issue on UWB Technology and Emerging Applications), for the Physical Communication Journal (Elsevier) (Special Issue on Advances in UWB Wireless Communications) and for the IEEE TRANSACTIONS ON Vehicular TeChNOLOGY (Special Session on indoor localization, tracking, and mapping with heterogeneous technologies). He served as an Editor for the IEEE TRANSACTIONS ON WIRELESS COMMUNICATIONS from 2006 to 2012 .

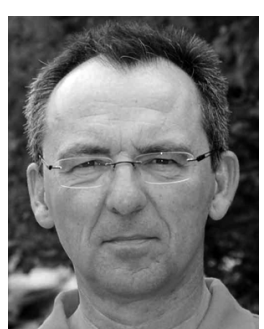

Luc Vandendorpe (F'06) was born in Mouscron, Belgium, in 1962. He received the electrical engineering degree (summa cum laude) and the Ph.D. degree from the Université Catholique de Louvain (UCL), Louvain-la-Neuve, Belgium, in 1985 and 1991, respectively. Since 1985 , he has been with the Communications and Remote Sensing Laboratory, UCL, where he first worked in the field of bit rate reduction techniques for video coding. In 1992, he was a Visiting Scientist and Research Fellow with the Telecommunications and Traffic Control Systems Group, Delft Technical University, The Netherlands, where he worked on spread spectrum techniques for personal communications systems. From October 1992 to August 1997, he was Senior Research Associate of the Belgian NSF at UCL, and an Invited Assistant Professor. Presently, he is Professor and Head of the Institute for Information and Communication Technologies, Electronics and Applied Mathematics. His current interest is in digital communication systems and more precisely resource allocation for OFDM(A)-based multicell systems, MIMO and distributed MIMO, sensor networks, turbo based communications systems, physical layer security, and UWB-based positioning. Dr. Vandendorpe was a co-recipient of the Biennal Alcatel-Bell Award from the Belgian NSF for a contribution in the field of image coding in 1990. In 2000 , he was a co-recipient (with J. Louveaux and F. Deryck) of the Biennal Siemens Award from the Belgian NSF for a contribution about filter bank based multicarrier transmission. In 2004, he was a co-winner (with J. Czyz) of the Face Authentication Competition, FAC 2004. He is or has been a TPC member for numerous IEEE conferences (VTC Fall, Globecom Communications Theory Symposium, SPAWC, ICC) and for the Turbo Symposium. He was Co-Technical Chair (with P. Duhamel) for IEEE ICASSP 2006. He was an editor of the IEEE TRANSACTIONS ON COMMUNICATIONS FOR SYNCHRONIZATION AND EQUALIZATION between 2000 and 2002, Associate Editor of the IEEE TRANSACTIONS ON WIRELESS COMMUNICATIONS between 2003 and 2005 , and Associate Editor of the IEEE TRAnsactions on Signal Processing between 2004 and 2006. He was Chair of the IEEE Benelux joint chapter on Communications and Vehicular Technology between 1999 and 2003. He was an elected member of the Signal Processing for Communications Committee between 2000 and 2005, and between 2009 and 2011, and an elected member of the Sensor Array and Multichannel Signal Processing Committee of the Signal Processing Society between 2006 and 2008. He is the Editor-in-Chief for the EURASIP Journal on Wireless Communications and Networking. 